\title{
PSEUDO-MARKOV SYSTEMS AND INFINITELY GENERATED SCHOTTKY GROUPS
}

\author{
BERND STRATMANN AND MARIUSZ URBAŃSKI
}

\begin{abstract}
In this paper we extend the theory of conformal graph directed Markov systems to what we call conformal pseudo-Markov systems. Unlike graph directed Markov systems, pseudo-Markov systems may have a countable infinite set of vertices as well as a countable infinite set of edges. After having developed appropriate symbolic dynamics for pseudo-Markov systems, we analyse these systems giving extensions of various aspects of the thermodynamical formalism and of fractal geometry. Most important, by establishing the existence of conformal measures along with their invariant versions, we obtain a generalised form of Bowen's formula for the Hausdorff dimension of the limit set of a conformal pseudo-Markov system, as well as a formula for the closure of the limit set. Finally, we apply our analysis to the theory of Kleinian groups. Here we obtain the existence of a rather exotic class of infinitely generated Schottky groups of the second kind (acting on $(d+1)$-dimensional hyperbolic space), containing groups with limit sets of Hausdorff dimension equal to any given number $t \leq d$, whereas their Poincaré exponent can be less than any given positive number $s<t$. Additionally, the dissipative part of the limit sets of these groups is shown to have further interesting properties.
\end{abstract}

\section{INTRODUCTION}

In this paper we extend the theory of conformal graph directed Markov systems to what we call conformal pseudo-Markov systems. These systems can have a countable infinite set of edges, and unlike graph directed Markov systems (see [9] for a fairly complete exposition), they may also have a countable infinite set of vertices. Additionally, certain distortion conditions are significantly weakend in the present paper. After having developed suitable symbolic dynamics for pseudo-Markov systems, we analyse these systems giving extensions of various aspects of the thermodynamical formalism and of fractal geometry. Most important, by establishing the existence of conformal measures along with their invariant versions, we obtain a generalised form of Bowen's formula [2] for the Hausdorff dimension of the limit set of a conformal pseudoMarkov system, as well as a formula for the closure of the limit set. Finally, we apply our formalism to the theory of Kleinian groups and obtain the existence of an interesting, rather exotic class of infinitely generated Schottky groups of the second kind. Note that there is a significant qualitative difference between pseudo-Markov systems with a countable infinite set of vertices and graph directed Markov systems, and the step from a finite to an infinite set of vertices is certainly much more demanding than the step from iterated function systems (one vertex) to graph directed Markov systems (finitely many vertices). Also, we expect

The research of the second author was supported in part by the NSF Grant DMS 0400481. 
that pseudo-Markov systems will develop into a powerful geometric theory with interesting applications in for instance complex dynamics and non-commutative geometry.

For our applications to Kleinian groups recall that Patterson [13], making use of a careful computational study of Poincaré series of free products of finitely generated Schottky groups acting on $(d+1)$-dimensional hyperbolic space $\mathbb{H}^{d+1}$, showed that there are infinitely generated Schottky groups of the first kind with critical exponent of their Poincaré series arbitrarily close to zero. By applying the theory of conformal pseudo-Markov systems developed in this paper, we extend this class of groups to infinitely generated Schottky groups $G$ of the second kind which have the properties that the Hausdorff dimension $\operatorname{HD}(L(G))$ of their limit sets $L(G)$ can be equal to any given number $t \in(0, d]$, whereas the critical exponent $\delta(G)$ of their Poincaré series can be less than any given positive number $s<t$. Moreover, these groups have the remarkable additional property that $\operatorname{HD}(L(G))$ is equal to the Hausdorff dimension of the set $\Delta(G)$ of accumulation points of the generating balls, whereas the complement within $L(G)$ of the $G$-orbit of $\Delta(G)$ has already Hausdorff dimension equal to $\delta(G)$.

Nevertheless, the main goal of this paper is the development of the theory of conformal pseudo-Markov systems. This development was originally motivated by the combinatorics and geometry of the type of Schottky groups just mentioned, where one should note that for these groups the theory of graph directed Markov systems as developed for instance in [8] and [9] is not applicable. More precisely, our analysis of conformal pseudo-Markov systems is structured as follows. In Section 2 we extend various aspects of the thermodynamical formalism to shift spaces generated by an infinite alphabet. Most notable, by studying the Perron-Frobenius operator and some relevant pressure functions, we obtain for these systems the existence of pseudo-conformal measures and their invariant versions. In Section 3 we introduce the concept of pseudo-Markov systems and give a first analysis of the limit sets of these systems. We then investigate certain families $F$ of functions and introduce the notion 'pseudo-conformality' for a pseudo-Markov systems. Using the results of Section 2, we eventually obtain for pseudo-conformal pseudo-Markov systems the existence of $F$-pseudoconformal measures under very weak conditions on the family $F$. Finally, in Section 4 we introduce conformal pseudo-Markov systems. After having discussed some of their main basic properties, especially their pseudo-conformality, we introduce the concept of thinness for a conformal pseudo-Markov system. Roughly speaking, thinness implies a rather rapid decay of the sizes of the generators. Our main results in Section 4 are a generalization of Bowen's Formula and the existence of suitable conformal measures. In particular, we obtain that for a thin conformal pseudo-Markov system the Hausdorff dimension of its limit set is equal to its hyperbolic dimension, that is the supremum of the Hausdorff dimensions of the limit sets of all finite subsystems.

Acknowledgement: We would like to thank I.H.E.S. at Bures-sur-Yvette for the warm hospitality and excellent working conditions while we started with the work towards this paper. 


\section{Thermodynamical Formalism for Systems with Countable Alphabets}

\subsection{Symbolic Dynamics.}

Throughout let $E$ be a countable alphabet which is either finite or infinite, and let

$$
A: E \times E \rightarrow\{0,1\}
$$

be some arbitrary function, called the incidence matrix. The set of infinite admissible words is given by

$$
E_{A}^{\infty}:=\left\{\omega \in E^{\infty}: A_{\omega_{i} \omega_{i+1}}=1 \text { for all } i \in \mathbb{N}\right\} .
$$

Let $E_{A}^{*}$ refer to the set of all finite admissable subwords of $E_{A}^{\infty}$. Also, the left shift map $\sigma: E_{A}^{\infty} \rightarrow E_{A}^{\infty}$ is given for $\omega_{1} \omega_{2} \ldots \in E_{A}^{\infty}$ by

$$
\sigma\left(\omega_{1} \omega_{2} \omega_{3} \ldots\right):=\omega_{2} \omega_{3} \ldots
$$

Occasionally, we will consider this shift to be defined on words of finite length. Given $\omega=$ $\omega_{1} \omega_{2} \ldots \omega_{n} \in E^{*}$, let $|\omega|:=n$ denote the length of the word $\omega$, and let $E_{A}^{n}$ refer to the set of all words in $E_{A}^{*}$ of length $n$. If $\omega=\omega_{1} \omega_{2} \ldots \in E_{A}^{\infty}$ and $n \in \mathbb{N}$, then

$$
\left.\omega\right|_{n}:=\omega_{1} \ldots \omega_{n} .
$$

refers to the initial segment of $\omega$ of length $n$. Finally, for $\tau \in E_{A}^{n}$ we define

$$
[\tau]:=\left\{\omega \in E_{A}^{\infty}:\left.\omega\right|_{n}=\tau\right\}
$$

Throughout the entire paper we will always assume that the matrix $A$ is finitely irreducible, that is there exists a finite set $\Xi \subset E_{A}^{*}$ such that for all $a, b \in E$ there exists $\alpha \in \Xi$ such that $a \alpha b \in E_{A}^{*}$.

\subsection{Topological Pressure on the Symbol Space.}

With the notation from the previous section, consider some arbitrary set $D \subseteq E$ and some arbitrary function $f: D_{A}^{\infty} \rightarrow \mathbb{R}$. For $n \in \mathbb{N}$, the $n$-th partition function $Z_{n}(D, f)$ is defined by

$$
Z_{n}(D, f):=\sum_{\omega \in D_{A}^{n}} \exp \left(\sup _{\tau \in[\omega] \cap D_{A}^{\infty}}\left(S_{n} f(\tau)\right)\right),
$$

where

$$
S_{n} f:=\sum_{j=0}^{n-1} f \circ \sigma^{j}
$$

In case $D=E$, then we simply write $Z_{n}(f)$ instead of $Z_{n}(E, f)$. One immediately verifies that the sequence $\left(\log Z_{n}(D, f)\right)_{n \in \mathbb{N}}$ is subadditive. This allows to define the topological pressure of $f$ with respect to the shift map $\sigma: D_{A}^{\infty} \rightarrow D_{A}^{\infty}$ by

$$
\mathrm{P}_{D}(f):=\lim _{n \rightarrow \infty} \frac{1}{n} \log Z_{n}(D, f)=\inf \left\{\frac{1}{n} \log Z_{n}(D, f): n \in \mathbb{N}\right\} .
$$

In case $D=E$, then we simply write $\mathrm{P}(f)$ instead of $P_{E}(f)$. Finally, we require the following notion of finite acceptability, which is weaker than the finite accepability introduced in [9]. 
Definition 2.1. A function $f: E_{A}^{\infty} \rightarrow \mathbb{R}$ is called finitely acceptable if and only if $f$ is uniformly continuous and

$$
\sup \left(\left.f\right|_{[e]}\right)-\inf \left(\left.f\right|_{[e]}\right)<\infty \text { for all } e \in E
$$

Note that the concept of acceptable functions considered in [9] is a slightly stronger. There we assumed additionally that $\sup \left\{\sup \left(\left.f\right|_{[e]}\right)-\inf \left(\left.f\right|_{[e]}\right): e \in E\right\}<\infty$.

Theorem 2.2. If $f: E_{A}^{\infty} \rightarrow \mathbb{R}$ is finitely acceptable, then

$$
\mathrm{P}(f)=\sup \left\{\mathrm{P}_{D}(f): D \subset E \text { finite }\right\}
$$

Proof. The inequality $\mathrm{P}(f) \geq \sup \left\{\mathrm{P}_{D}(f)\right\}$ is obvious. In order to prove the reverse inequality, first suppose that $\mathrm{P}(f)<\infty$. Put $q:=\# \Lambda, p:=\max \{|\omega|: \omega \in \Xi\}$ and $T:=\min \left\{\inf \left(S_{|\omega|} f\right): \omega \in \Xi\right\}$. Let $\epsilon>0$ be fixed. Since $f$ is uniformly continuituous, there exists $l \in N$ such that $|f(\omega)-f(\tau)|<\epsilon$, for all $\omega, \tau \in E_{A}^{\infty}$ with $\left.\omega\right|_{l}=\left.\tau\right|_{l}$. Now, let $k \in \mathbb{N}$ be fixed. By subadditivity of the sequence $\left(\log Z_{n}(D, f)\right)_{n \in \mathbb{N}}$, we have that $\frac{1}{k} \log Z_{k}(f) \geq \mathrm{P}(f)$. Also, note that there exists a finite set $D \subset E$ such that

$$
\frac{1}{k} \log Z_{k}(D, f)>\mathrm{P}(f)-\epsilon
$$

Without loss of generality we can assume that $D \subseteq \Xi$. Since $f$ is finitely acceptable, it follows

$$
M_{D}:=\max \left\{\sup \left(\left.f\right|_{[e]}\right)-\inf \left(\left.f\right|_{[e]}\right): e \in D\right\}<\infty
$$

Now, for each $\tau=\tau_{1} \tau_{2} \ldots \tau_{n} \in\left(D_{A}^{k}\right)^{n}$ there exist elements $\alpha_{1}, \alpha_{2}, \ldots, \alpha_{n-1} \in \Xi$ such that $\bar{\tau}=\tau_{1} \alpha_{1} \tau_{2} \alpha_{2} \ldots \tau_{n-1} \alpha_{n-1} \tau_{n} \in E_{A}^{*}$. Note that the function given by $\tau \mapsto \bar{\tau}$ is at most $q^{n-1}$ to 
1. We then have for each $n \in \mathbb{N}$,

$$
\begin{aligned}
q^{n-1} \sum_{i=k n}^{k n+p(n-1)} Z_{i}(F, f) & \geq \sum_{\tau \in\left(D_{A}^{k}\right)^{n}} \exp \left(\sup _{[\bar{\tau}: D]} \sum_{j=0}^{|\bar{\tau}|} f \circ \sigma^{j}\right) \\
& \geq \sum_{\tau \in\left(D_{A}^{k}\right)^{n}} \exp \left(\inf _{[\bar{\tau}]}^{|\bar{\tau}|} \sum_{j=0} f \circ \sigma^{j}\right) \\
& \geq \sum_{\tau \in\left(D_{A}^{k}\right)^{n}} \exp \left(\left.\sum_{i=1}^{n} \inf \left(S_{k}(f)\right)\right|_{\left[\tau_{i}\right]}+T(n-1)\right) \\
& =\left.\exp (T(n-1)) \sum_{\tau \in\left(D_{A}^{k}\right)^{n}} \exp \sum_{i=1}^{n} \inf \left(S_{k}(f)\right)\right|_{\left[\tau_{i}\right]} \\
& \geq \exp (T(n-1)) \sum_{\tau \in\left(D_{A}^{k}\right)^{n}} \exp \left(\sum_{i=1}^{n}\left(\left.\sup \left(S_{k}(f)\right)\right|_{\left[\tau_{i}\right]}-(k-l) \epsilon-M_{D} l\right)\right) \\
& =\left.\exp \left(T(n-1)-(k-l) \epsilon n-M_{D} l n\right) \sum_{\tau \in\left(D_{A}^{k}\right)^{n}} \exp \sum_{i=1}^{n} \sup \left(S_{k}(f)\right)\right|_{\left[\tau_{i}\right]} \\
& =\mathrm{e}^{-T} \exp \left(n\left(T-(k-l) \epsilon-M_{D} l\right)\right)\left(\sum_{\tau \in\left(D_{A}^{k}\right)} \exp \left(\left.\sup \left(S_{k}(f)\right)\right|_{[\tau]}\right)\right)^{n}
\end{aligned}
$$

Hence, there exists a sequence $\left(i_{n}\right)_{n \in \mathbb{N}}$ such that $k n \leq i_{n} \leq(k+p) n$ and

$$
Z_{i_{n}}(D, f) \geq \frac{1}{p n} \mathrm{e}^{-T} \exp \left(n\left(T-(k-l) \epsilon-M_{D} l-\log q\right)\right) Z_{k}(D, f)^{n} .
$$

Therefore, using (2.2), we obtain for $k$ sufficiently large,

$$
\mathrm{P}_{D}(f)=\lim _{n \rightarrow \infty} \frac{1}{i_{n}} \log Z_{i_{n}}(D, f) \geq \frac{-|T|}{k}-\epsilon+\frac{l \epsilon}{k+p}-\frac{M_{D} l+\log p}{k}+\mathrm{P}(f)-2 \epsilon \geq \mathrm{P}(f)-7 \epsilon .
$$

By letting $\epsilon$ tend to 0 , the assertion follows for the case $\mathrm{P}(f)<\infty$. The case $\mathrm{P}(f)=\infty$ is obtained in a similar way, and we omit its proof.

\subsection{Perron-Frobenius Operators and Pseudo-Conformal Measures.}

Throughout this section let $f: E_{A}^{\infty} \rightarrow \mathbb{R}$ be a finitely acceptable function, and assume that $f$ is summable in the following sense.

Definition 2.3. A function $f: E_{A}^{\infty} \rightarrow \mathbb{R}$ is called summable if

$$
\sum_{e \in E} \exp \left(\sup \left(\left.f\right|_{[e]}\right)\right)<\infty
$$


With $C_{b}\left(E_{A}^{\infty}\right)$ referring to the space of bounded continuous functions equipped with the supremums norm $\|\cdot\|_{\infty}$, the Perron-Frobenius operator $\mathcal{L}_{f}: C_{b}\left(E_{A}^{\infty}\right) \rightarrow C_{b}\left(E_{A}^{\infty}\right)$ is defined for $g \in C_{b}\left(E_{A}^{\infty}\right)$ and $\omega=\omega_{1} \omega_{2} \ldots \in E_{A}^{\infty}$ by

$$
\mathcal{L}_{f}(g)(\omega):=\sum_{\substack{e \in E \\ A_{e \omega_{1}}=1}} \exp f(e \omega) g(e \omega) .
$$

Note that the summability of $f$ gives that $\left\|\mathcal{L}_{f}\right\|_{\infty} \leq \sum_{e \in E} \exp \left(\sup \left(\left.f\right|_{[e]}\right)\right)<\infty$. Also, for each $n \in \mathbb{N}$ we have

$$
\mathcal{L}_{f}^{n}(g)(\omega)=\sum_{\substack{\tau \in E_{A}^{n} \\ A_{\tau_{n} \omega_{1}}=1}} \exp \left(S_{n} f(\tau \omega)\right) g(\tau \omega) .
$$

The dual operator $\mathcal{L}_{f}^{*}: C_{b}^{*}\left(E_{A}^{\infty}\right) \rightarrow C_{b}^{*}\left(E_{A}^{\infty}\right)$ is given by

$$
\mathcal{L}_{f}^{*}(\mu)(g)=\mu\left(\mathcal{L}_{f}(g)\right)=\int \mathcal{L}_{f}(g) d \mu .
$$

Throughout this section we will always assume that $\tilde{m}$ is an $f$-pseudo-conformal measure. This means by definition that $\tilde{m}$ is an eigenmeasure of $\mathcal{L}_{f}^{*}$ which is in particular a Borel probability measure on $E_{A}^{\infty}$. Since $\mathcal{L}_{f}$ is a positive operator, we have for the eigenvalue $\lambda=\lambda(f)$ associated with $\tilde{m}$ that $\lambda \geq 0$. Also, since $\mathcal{L}_{f}^{* n}(\tilde{m})=\lambda^{n} \tilde{m}$, we clearly have for each $g \in C_{b}\left(E_{A}^{\infty}\right)$,

$$
\int \sum_{\substack{\tau \in E_{A}^{n} \\ A_{\tau_{n} \omega_{1}=1}}} \exp \left(S_{n} f(\tau \omega)\right) g(\tau \omega) d \tilde{m}(\omega)=\lambda^{n} \int g d \tilde{m} .
$$

Note that (2.4) extends immediately to the space of all bounded Borel functions on $E_{A}^{\infty}$. Let $\omega \in E_{A}^{n}$, let $B \subset E_{A}^{\infty}$ be some Borel set such that $A_{\omega_{n} \tau_{1}}=1$, for every $\tau=\tau_{1} \tau_{2} \ldots \in B$, and consider $g:=\mathbb{1}_{\omega B}$. Then (2.4) implies

$$
\begin{aligned}
\lambda^{n} \tilde{m}(\omega B) & =\int \sum_{\substack{\tau \in E_{A}^{n} \\
A_{\tau_{n} \rho_{1}}=1}} \exp \left(S_{n} f(\tau \rho)\right) \mathbb{1}_{\omega B}(\tau \rho) d \tilde{m}(\rho) \\
& =\int_{\left\{\rho \in B: A_{\omega_{n} \rho_{1}}=1\right\}} \exp \left(S_{n} f(\omega \rho)\right) d \tilde{m}(\rho) \\
& =\int_{B} \exp \left(S_{n} f(\omega \rho)\right) d \tilde{m}(\rho) .
\end{aligned}
$$

The following lemmata will turn out to be useful.

Lemma 2.4. We have that $\tilde{m}([e])>0$, for each $e \in E$.

Proof. Since $E_{A}^{\infty}=\bigcup_{e \in E}[e]$, there exists $a \in E$ such that $\tilde{m}([a])>0$. Since $A$ is irreducible, for each $e \in E$ there exists $\alpha \in E^{*}$ such that e $\alpha a \in E_{A}^{*}$. It then follows from (2.5) that

$$
\tilde{m}([e]) \geq \tilde{m}([e \alpha a])=\lambda^{-n} \int_{[a]} \exp \left(S_{n} f(e \alpha \rho)\right) d \tilde{m}(\rho)>0 .
$$


Lemma 2.5. If $f: E_{A}^{\infty} \rightarrow \mathbb{R}$ is summable and finitely acceptable, then $\lambda(f)=e^{\mathrm{P}(f)}$.

Proof. In order to show that $\log \lambda \leq \mathrm{P}(f)$, we apply (2.4) for $g=\mathbb{1}$ and obtain, for each $n \in \mathbb{N}$,

$$
\begin{aligned}
\lambda^{n} & =\sum_{\tau \in E_{A}^{n}} \sum_{\substack{e \in E \\
A_{\tau_{n} e=1}}} \int_{[e]} \exp \left(S_{n} f(\tau \omega)\right) d \tilde{m}(\omega) \\
& \leq \sum_{\tau \in E_{A}^{n}} \sum_{e \in E} \exp \left(\sup \left(\left.S_{n} f\right|_{[\tau]}\right)\right) \tilde{m}([e]) \\
& \leq \sum_{\tau \in E_{A}^{n}} \exp \left(\sup \left(\left.S_{n} f\right|_{[\tau]}\right)\right)=Z_{n}(f) .
\end{aligned}
$$

Inserting this estimate into the definition of $\mathrm{P}(f)$, the assertion follows.

In order to show that $\log \lambda \geq \mathrm{P}(f)$, let $D \subset E$ be finite. Observe that by Lemma 2.4 we have

$$
Q_{D}:=\min \{\tilde{m}([j]): j \in D\}>0 .
$$

Therefore, an application of (2.4) with $g=\mathbb{1}$ gives for each $n \in \mathbb{N}$, with $M_{D}$ defined as in the proof of Theorem 2.2,

$$
\begin{aligned}
\lambda^{n} & \geq \sum_{\tau \in D_{A}^{n}} \sum_{\substack{e \in D \\
A_{\tau_{n} e=1}}} \int_{[e]} \exp \left(S_{n} f(\tau \omega)\right) d \tilde{m}(\omega) \\
& \geq \sum_{\tau \in D_{A}^{n}} \exp \left(-M_{D}\right) \sum_{\substack{e \in D \\
A_{\tau_{n} e=1}}} \int_{[e]} \exp \left(\sup \left(S_{n} f\right)\right) d \tilde{m} \\
& =\sum_{\tau \in D_{A}^{n}} \exp \left(-M_{D}\right) \sum_{\substack{e \in D \\
A_{\tau_{n} e=1}}} \exp \left(\sup \left(\left.S_{n} f\right|_{[\tau]}\right)\right) \tilde{m}([e]) \\
& \geq \exp \left(-M_{D}\right) Q_{D} \sum_{\tau \in D_{A}^{n}} \sum_{\tau \in D_{A}^{n}} \exp \left(\sup \left(\left.S_{n} f\right|_{[\tau]}\right)\right) \\
& =\exp \left(-M_{D}\right) Q_{D} Z_{n}(f, D) .
\end{aligned}
$$

It follows that $\log \lambda \geq \mathrm{P}(f)$, and by inserting this into the definition of $\mathrm{P}(f)$, the assertion follows.

Our aim is to show that a pseudo-conformal measure exists already under very mild conditions. We start with the following well-known result which is essentially due to Bowen ([2]).

Lemma 2.6. If the alphabet $E$ is finite and if $f: E_{A}^{\infty} \rightarrow \mathbb{R}$ is continuous (and hence summable and finitely acceptable), then there exists an $f$-pseudo-conformal measure.

Proof. Consider the map given by

$$
\mu \mapsto \frac{\mathcal{L}_{f}^{*}(\mu)}{\mathcal{L}_{f}^{*}(\mu)(\mathbb{1})} .
$$


Clearly, this is a continuous automorphism of the compact convex space of all Borel probability measures on $E_{A}^{\infty}$ into itself. The Schauder-Tychonov Theorem then guarantees the existence of a fixed point, and one immediately verifies that this fixed point is an $f$-pseudo-conformal measure.

With these preparations we now turn to the main result of this section. Note that the proof of the following theorem is similar to the proof of the corresponding result for graph directed Markov systems in [9] (Theorem 2.7.3).

Theorem 2.7. Every finitely acceptable and summable function $f: E_{A}^{\infty} \rightarrow \mathbb{R}$ gives rise to an $f$-pseudo-conformal measure.

Proof. For ease of exposition we can assume without loss of generality that $E=\mathbb{N}$. Since $A$ is finitely irreducible, there exists a strictly increasing sequence $\left\{D_{n}\right\}_{n \in \mathbb{N}}$ of finite sets $D_{n} \subset E$ such that

$$
\bigcup_{n=1}^{\infty} D_{n}=E \text { and } D_{n}^{*} \supset \Xi \text {, for all } n \in \mathbb{N} \text {. }
$$

Clearly, the incidence matrix restricted to $D_{n} \times D_{n}$ is irreducible, for each $n \in \mathbb{N}$. By Lemma 2.6 we then have that there exists an eigenmeasure $\tilde{m}_{n}$ of the conjugate $\mathcal{L}_{n}^{*}$ of the Perron-Frobenius operator

$$
\mathcal{L}_{n}: C\left(\left(D_{n}\right)_{A}^{\infty}\right) \rightarrow C\left(\left(D_{n}\right)_{A}^{\infty}\right)
$$

associated to the function $\left.f\right|_{\left(D_{n}\right)_{A}^{\infty}}$. Occasionally, we will view $\mathcal{L}_{n}$ as acting on $C\left(E_{A}^{\infty}\right)$, and $\mathcal{L}_{n}^{*}$ as acting on $C^{*}\left(E_{A}^{\infty}\right)$. Now, our first aim is to obtain tightness of the sequence $\left\{\tilde{m}_{n}\right\}_{n \in \mathbb{N}}$ of Borel probability measures on $E_{A}^{\infty}$. For this let $\mathrm{P}_{n}:=\mathrm{P}\left(\left.\sigma\right|_{\left(D_{n}\right)_{A}^{\infty}},\left.f\right|_{\left(D_{n}\right)_{A}^{\infty}}\right)$. Obviously, we have $\mathrm{P}_{n} \geq \mathrm{P}_{1}$ for all $n \in \mathbb{N}$. Let $\pi_{k}: E_{A}^{\infty} \rightarrow E$ be the projection onto the $k$-th coordinate, that is $\pi_{k}\left(e_{1} e_{2} \ldots\right)=e_{k}$ for each $k \in \mathbb{N}$. By Lemma $2.5, e^{\mathrm{P}_{n}}$ is the eigenvalue of $\mathcal{L}_{n}^{*}$ corresponding to the eigenmeasure $\tilde{m}_{n}$. Therefore, by applying (2.5), we obtain for each $n, k \in N$ and every $e \in E$,

$$
\begin{aligned}
\tilde{m}_{n}\left(\pi_{k}^{-1}(e)\right) & =\sum_{\substack{\omega \in\left(D_{n}\right)_{A}^{k} \\
\omega_{k}=e}} \tilde{m}_{n}([\omega]) \leq \sum_{\substack{\omega \in\left(D_{n}\right)_{A}^{k} \\
\omega_{k}=e^{k}}} \exp \left(\sup \left(\left.S_{k} f\right|_{[\omega]}\right)-\mathrm{P}_{n} k\right) \\
& \leq \mathrm{e}^{-\mathrm{P}_{n} k} \sum_{\substack{\omega \in\left(D_{n}\right)_{A}^{k} \\
\omega_{k}=e^{k}}} \exp \left(\sup \left(\left.S_{k-1} f\right|_{[\omega]}\right)+\sup \left(\left.f\right|_{[e]}\right)\right) \\
& \leq \mathrm{e}^{-\mathrm{P}_{1} k}\left(\sum_{i \in E} \mathrm{e}^{\sup \left(\left.f\right|_{[i]}\right)}\right)^{k-1} \mathrm{e}^{\sup \left(\left.f\right|_{[e]}\right)} .
\end{aligned}
$$

Therefore,

$$
\tilde{m}_{n}\left(\pi_{k}^{-1}([e+1, \infty))\right) \leq \mathrm{e}^{-\mathrm{P}_{1} k}\left(\sum_{i \in E} \mathrm{e}^{\sup \left(\left.f\right|_{[i]}\right)}\right) \sum_{j>e}^{k-1} \mathrm{e}^{\sup \left(\left.f\right|_{[j]}\right)}
$$


Now, let $\epsilon>0$ be fixed. For each $k \in \mathbb{N}$, let $n_{k} \in \mathbb{N}$ be chosen such that

$$
\mathrm{e}^{-\mathrm{P}_{1} k}\left(\sum_{i \in E} \mathrm{e}^{\sup \left(\left.f\right|_{[i]}\right)}\right)^{k-1} \sum_{j>n_{k}} \mathrm{e}^{\sup \left(\left.f\right|_{[j]}\right)} \leq \frac{\epsilon}{2^{k}} .
$$

Then $\tilde{m}_{n}\left(\pi_{k}^{-1}\left(\left[n_{k}+1, \infty\right)\right)\right) \leq \epsilon / 2^{k}$, for all $n, k \in \mathbb{N}$, and hence,

$$
\tilde{m}_{n}\left(E_{A}^{\infty} \cap \prod_{k \in \mathbb{N}}\left[1, n_{k}\right]\right) \geq 1-\sum_{k \in \mathbb{N}} \tilde{m}_{n}\left(\pi_{k}^{-1}\left(\left[n_{k}+1, \infty\right)\right)\right) \geq 1-\sum_{k \in \mathbb{N}} \frac{\epsilon}{2^{k}}=1-\epsilon .
$$

Since $E_{A}^{\infty} \cap \prod_{k \in \mathbb{N}}\left[1, n_{k}\right]$ is a compact subset of $E_{A}^{\infty}$, it follows that the sequence $\left\{\tilde{m}_{n}\right\}_{n \in \mathbb{N}}$ is tight. We can now apply Prokhorov's Theorem, which gives that there exists a weak-limit point $\tilde{m}$ of the sequence $\left\{\tilde{m}_{n}\right\}_{n \in \mathbb{N}}$. In order to show that $\mathcal{L}_{f}^{*} \tilde{m}=\mathrm{e}^{\mathrm{P}(f)} \tilde{m}$, let $g \in C_{b}\left(E_{A}^{\infty}\right)$ and $\epsilon>0$ be fixed. Also, define the normalized Perron-Frobenius operator $\mathcal{L}_{0}:=\mathrm{e}^{-\mathrm{P}(f)} \mathcal{L}_{f}$. For the remainder of the proof let us assume that $n \in \mathbb{N}$ is chosen sufficiently large such that the following four inequalities are satisfied.

$$
\begin{gathered}
\sum_{i>n}\|g\|_{0} \exp \left(\sup \left(\left.f\right|_{[i]}\right)-\mathrm{P}(f)\right) \leq \frac{\epsilon}{6} \\
\sum_{i \in E}\|g\|_{0} \exp \left(\sup \left(\left.f\right|_{[i]}\right)\right) \mathrm{e}^{-\mathrm{P}_{1}}\left|e^{\mathrm{P}(f)}-e^{\mathrm{P}_{n}}\right| \leq \frac{\epsilon}{6}, \\
\left|\tilde{m}_{n}(g)-\tilde{m}(g)\right| \leq \frac{\epsilon}{3}
\end{gathered}
$$

and

$$
\left|\int \mathcal{L}_{0}(g) d \tilde{m}-\int \mathcal{L}_{0}(g) d \tilde{m}_{n}\right| \leq \frac{\epsilon}{3}
$$

Note that (2.7) is satisfied, since by Theorem 2.2 we have that $\lim _{n \rightarrow \infty} \mathrm{P}_{n}=\mathrm{P}(f)$. For $n$ in this range, we define $g_{n}:=\left.g\right|_{\left(D_{n}\right)_{A}^{\infty}}$ and consider the normalized Perron-Frobenius operator $\mathcal{L}_{0, n}:=\mathrm{e}^{-\mathrm{P}_{n}} \mathcal{L}_{n}$. Let us make the following two observations.

$$
\begin{aligned}
\mathcal{L}_{0, n}^{*} \tilde{m}_{n}(g) & =\int_{E^{\infty}} \sum_{\substack{i \leq n \\
A_{i \omega_{n}}=1}} g(i \omega) \exp \left(f(i \omega)-\mathrm{P}_{n}\right) d \tilde{m}_{n}(\omega) \\
& =\int_{\left(D_{n}\right)_{A}^{\infty}} \sum_{\substack{i \leq n \\
A_{i \omega_{n}}=1}} g(i \omega) \exp \left(f(i \omega)-\mathrm{P}_{n}\right) d \tilde{m}_{n}(\omega) \\
& =\int_{\left(D_{n}\right)_{A}^{\infty}} \sum_{\substack{i \leq n \\
A_{i \omega_{n}}=1}} g_{n}(i \omega) \exp \left(f(i \omega)-\mathrm{P}_{n}\right) d \tilde{m}_{n}(\omega) \\
& =\mathcal{L}_{0, n}^{*} \tilde{m}_{n}\left(g_{n}\right)=\tilde{m}_{n}\left(g_{n}\right)
\end{aligned}
$$

and

$$
\tilde{m}_{n}\left(g_{n}\right)-\tilde{m}_{n}(g)=\int_{\left(D_{n}\right)_{A}^{\infty}}\left(g_{n}-g\right) d \tilde{m}_{n}=\int_{\left(D_{n}\right)_{A}^{\infty}} 0 d \tilde{m}_{n}=0 .
$$


Hence, using the triangle inequality, it follows

$$
\begin{aligned}
\left|\mathcal{L}_{0}^{*} \tilde{m}(g)-\tilde{m}(g)\right| & \leq\left|\mathcal{L}_{0}^{*} \tilde{m}(g)-\mathcal{L}_{0}^{*} \tilde{m}_{n}(g)\right|+\left|\mathcal{L}_{0}^{*} \tilde{m}_{n}(g)-\mathcal{L}_{0, n}^{*} \tilde{m}_{n}(g)\right|+ \\
& +\left|\mathcal{L}_{0, n}^{*} \tilde{m}_{n}(g)-\tilde{m}_{n}\left(g_{n}\right)\right|+\left|\tilde{m}_{n}\left(g_{n}\right)-\tilde{m}_{n}(g)\right|+\left|\tilde{m}_{n}(g)-\tilde{m}(g)\right| .
\end{aligned}
$$

In here we obtain for the second summand, by applying (2.6) and (2.7),

$$
\begin{aligned}
\mid \mathcal{L}_{0}^{*} \tilde{m}_{n}(g) & -\mathcal{L}_{0, n}^{*} \tilde{m}_{n}(g) \mid= \\
& =\mid \int_{E_{A}^{\infty}} \sum_{\substack{i \leq n \\
A_{i \omega_{n}=1}}} g(i \omega)\left(\exp (f(i \omega)-\mathrm{P}(f))-\exp \left(f(i \omega)-\mathrm{P}_{n}\right)\right) d \tilde{m}_{n}(\omega) \\
& +\int_{E_{A}^{\infty}} \sum_{\substack{i>n \\
A_{i \omega_{n}}=1}} g(i \omega) \exp (f(i \omega)-\mathrm{P}(f)) d \tilde{m}_{n}(\omega) \mid \\
& \leq \sum_{i \leq n}\|g\|_{0} \mathrm{e}^{f(i \omega)} \mathrm{e}^{-\mathrm{P}_{n}}\left|e^{\mathrm{P}(f)}-e^{\mathrm{P}_{n}}\right|+\sum_{i>n}\|g\|_{0} \exp \left(\sup \left(\left.f\right|_{[i]}\right)-\mathrm{P}(f)\right) \\
& \leq \sum_{i \in E}\|g\|_{0} \exp \left(\sup \left(\left.f\right|_{[i]}\right)\right) \mathrm{e}^{-\mathrm{P}_{1}}\left|e^{\mathrm{P}(f)}-e^{\mathrm{P}_{n}}\right|+\frac{\epsilon}{6} \\
& \leq \frac{\epsilon}{6}+\frac{\epsilon}{6}=\frac{\epsilon}{3} .
\end{aligned}
$$

Combining (2.12) with (2.8), (2.9), (2.10), (2.11) and (2.13), it now follows that

$$
\left|\mathcal{L}_{0}^{*} \tilde{m}(g)-\tilde{m}(g)\right| \leq \frac{\epsilon}{3}+\frac{\epsilon}{3}+\frac{\epsilon}{3}=\epsilon .
$$

By letting $\epsilon$ tend to 0 , we conclude that $\mathcal{L}_{0}^{*} \tilde{m}(g)=\tilde{m}(g)$, or alternatively $\mathcal{L}_{f}^{*} \tilde{m}(g)=\mathrm{e}^{\mathrm{P}(f)} \tilde{m}(g)$. Since $g \in C_{b}\left(E_{A}^{\infty}\right)$ was assumed to be arbitrary, we have now shown that $\mathcal{L}_{f}^{*} \tilde{m}=\mathrm{e}^{\mathrm{P}(f)} \tilde{m}$.

Definition 2.8. A summable and finitely acceptable function $f: E_{A}^{\infty} \rightarrow \mathbb{R}$ is called boundedly distorted if and only if there exists a constant $Q>0$ such that

$$
\text { if }\left.\tau\right|_{n+1}=\left.\omega\right|_{n+1} \text { for some } \omega, \tau \in E_{A}^{\infty} \text { and } n \in \mathbb{N} \text {, then }\left|S_{n} f(\omega)-S_{n} f(\tau)\right| \leq Q \text {. }
$$

Also, we require the following notation. If $\omega=\omega_{1} \ldots \omega_{n} \in E_{A}^{n}$ for some $n \in \mathbb{N}$, then we put

$$
\Sigma_{\omega}^{-}:=\left\{\tau \in E_{A}^{\infty}: A_{\omega_{n} \tau_{1}}=1\right\}
$$

and let $\sigma_{\omega}^{-n}: \Sigma_{\omega}^{-} \rightarrow E_{A}^{\infty}$ refer to the map which is given for $\tau \in \Sigma_{\omega}^{-}$by

$$
\sigma_{\omega}^{-n}(\tau):=\omega \tau \text {. }
$$

Remark 2.9. Note that the notion 'boundedly distorted' represents a slightly weaker condition than the type of bounded distortion used in connection with graph directed Markov systems. More precisely, for the analysis of graph directed Markov systems in [9] it was sufficient to assume that $\left|S_{n} f(\omega)-S_{n} f(\tau)\right| \leq Q$ as long as the first $n$ entries of $\tau$ and $\omega$ coincide. Whereas 
here, where we allow an infinite set of vertices, it is necessary to demand that $\tau$ and $\omega$ coincide at their first $n+1$ entries.

The following theorem gives the final result of this section. We remark that a similar result was obtained in [9] for graph directed Markov systems. The difference is that here we do not necessarily have that the Radon-Nikodym derivative of $\tilde{m}$ with respect to $\tilde{\mu}$ is bounded from below and above.

Theorem 2.10. Let $\tilde{m}$ be an f-pseudo-conformal measure, for some summable, finitely acceptable and boundedly distorted function $f: E_{A}^{\infty} \rightarrow \mathbb{R}$. Then there exists a shift-invariant Borel probability measure $\tilde{\mu}$ which is equivalent to $\tilde{m}$.

Proof. Fix $e \in E, n \in \mathbb{N}$, and $\tau=\tau_{1} \ldots \tau_{n} \in E_{A}^{n}$ such that $A_{\tau_{n} e}=1$. Since $\tilde{m}$ is $f$-pseudo-conformal, it follows for each Borel set $B \subseteq[e]$,

$$
\tilde{m}\left(\sigma_{\tau}^{-n}(B)\right)=\int_{B} \exp \left(S_{n} f(\tau \rho)-\mathrm{P}(f) n\right) d \tilde{m}(\rho) \leq e^{Q} \exp \left(\inf \left(\left.S_{n} f\right|_{[\tau e]}\right)\right) \tilde{m}(B)
$$

and

$$
\tilde{m}\left(\sigma_{\tau}^{-n}(B)\right)=\int_{B} \exp \left(S_{n} f(\tau \rho)-\mathrm{P}(f) n\right) d \tilde{m}(\rho) \geq \exp \left(\inf \left(\left.S_{n} f\right|_{[\tau e]}\right)\right) \tilde{m}(B) .
$$

If in particular $B=[e]$, then $(2.15)$ reads as $\tilde{m}\left(\sigma_{\tau}^{-n}([e])\right) \leq e^{Q} \exp \left(\inf \left(\left.S_{n} f\right|_{\left[\tau^{-n}([e]\right.}\right)\right) \tilde{m}([e])$. Therefore, in this case we have

$$
\exp \left(\inf \left(\left.S_{n} f\right|_{\left[\tau^{-n}([e])\right.}\right)\right) \geq e^{-Q} \frac{\tilde{m}\left(\sigma_{\tau}^{-n}([e])\right)}{\tilde{m}([e])}=e^{-Q} \frac{\tilde{m}([\tau e])}{\tilde{m}([e])} .
$$

Similarly, using (2.16), we obtain

$$
\exp \left(\inf \left(\left.S_{n} f\right|_{\left[\tau^{-n}([e])\right.}\right)\right) \leq \frac{\tilde{m}([\tau e])}{\tilde{m}([e])} .
$$

Inserting (2.18) into (2.15) and (2.17) into (2.16), we obtain

$$
\tilde{m}\left(\sigma_{\tau}^{-n}(B)\right) \leq \frac{e^{Q}}{\tilde{m}([e])} \tilde{m}([\tau e]) \tilde{m}(B)
$$

and

$$
\tilde{m}\left(\sigma_{\tau}^{-n}(B)\right) \geq \frac{e^{-Q}}{\tilde{m}([e])} \tilde{m}([\tau e]) \tilde{m}(B) .
$$

By summing (2.19) and (2.20) over all $\tau \in E_{A}^{n}$ for which $A_{\tau_{n} e}=1$, we conclude that

$$
\frac{e^{-Q}}{\tilde{m}([e])} \tilde{m}([\tau e]) \tilde{m}(B) \leq \tilde{m}\left(\sigma^{-n}(B)\right) \leq \frac{e^{Q}}{\tilde{m}([e])} \tilde{m}([\tau e]) \tilde{m}(B) .
$$


Now, with $L$ referring to a Banach limit defined on the Banach space of all bounded sequences of real numbers, let $\tilde{\mu}$ be defined by $\tilde{\mu}(A):=L\left(\left(\tilde{m}\left(\sigma^{-n}(A)\right)\right)_{n \in \mathbb{N}}\right)$, for each Borel set $A \subseteq$ $E_{A}^{\infty}$. One immediately verifies that $\tilde{\mu}$ is a finite, non-trivial, $\sigma$-invariant and finitely additive function defined on the $\sigma$-algebra of all Borel sets of $E_{A}^{\infty}$. Furthermore, using (2.21), we obtain for all $e \in E$ and arbitrary Borel sets $B \subset[e]$,

$$
\frac{e^{-Q}}{\tilde{m}([e])} \tilde{m}([\tau e]) \tilde{m}(B) \leq \tilde{\mu}(B) \leq \frac{e^{Q}}{\tilde{m}([e])} \tilde{m}([\tau e]) \tilde{m}(B) .
$$

Hence, using the fact that $\tilde{m}$ is a countably additive measure, it follows that $\tilde{\mu}$ must also be countably additive. This finishes the proof.

\section{Pseudo-Markov Systems}

\subsection{Preliminaries for Pseudo-Markov Systems.}

In this section we introduce the concept 'pseudo-Markov system' and then give a first analysis of the limit sets of these systems. Note that pseudo-Markov systems may have an infinite set of vertices, and therefore these systems represent a significant extension of graph directed Markov systems. As in the previous section, we always assume that $E$ is a countable alphabet, which is either finite or infinite, and that $A: E \times E \rightarrow\{0,1\}$ is some given finitely irreducible incidence matrix.

Definition 3.1. Let $(Y, \rho)$ be a bounded metric space and let $\left\{X_{e}: e \in E\right\}$ be a family of compact subsets of $Y$. A set $\mathcal{S}=\left\{\phi_{e}: e \in E\right\}$ of continuous injections $\phi_{e}: X_{e} \rightarrow Y$ is called $a$ pseudo-Markov system if the following conditions are satisfied.

(a) The maps in $\mathcal{S}$ are uniform contractions. That is, there exists a constant $0<s<1$ such that

$$
\rho\left(\phi_{e}(y), \phi_{e}(x)\right) \leq s \rho(y, x) \text { for all } e \in E, x, y \in X_{e} .
$$

(b) (Open Set Condition) If $a, b \in E$ and $a \neq b$, then

$$
\phi_{a}\left(\operatorname{Int}\left(X_{a}\right)\right) \cap \phi_{b}\left(\operatorname{Int}\left(X_{b}\right)\right)=\emptyset .
$$

(c) (Markov Property) If $a, b \in E$, then

$$
\text { either } \left.\phi_{a}\left(\operatorname{Int}\left(X_{a}\right)\right) \cap \operatorname{Int}\left(X_{b}\right)\right)=\emptyset \text { or } \phi_{a}\left(X_{a}\right) \subset X_{b} \text {. }
$$

(d) If $a, b \in E$ such that $A_{a b}=1$, then

$$
\phi_{b}\left(X_{b}\right) \subset X_{a} .
$$

For a finite word $\omega=\omega_{1} \omega_{2} \ldots \omega_{n} \in E_{A}^{*}$, we write

$$
\phi_{\omega}:=\phi_{\omega_{1}} \circ \phi_{\omega_{2}} \ldots \circ \phi_{\omega_{n}}: X_{\omega_{n}} \rightarrow \phi_{\omega_{1}}\left(X_{\omega_{1}}\right) .
$$


In order to define the limit set of a pseudo-Markov system $\mathcal{S}$, observe that by condition (d) in Definition 3.1, we have for $\omega=\omega_{1} \omega_{2} \ldots \in E_{A}^{\infty}$ that the sequence $\left(\phi_{\left.\omega\right|_{n}}\left(X_{\omega_{n}}\right)\right)_{n \in \mathbb{N}}$ is a descending family of compact subsets of $Y$. Also, by condition (a) in Definition 3.1, we have $\lim _{n \rightarrow \infty} \operatorname{diam}\left(\phi_{\left.\omega\right|_{n}}\left(X_{\omega_{n}}\right)\right)=0$. Combining these two observations, it follows that the intersection $\bigcap_{n=1}^{\infty} \phi_{\left.\omega\right|_{n}}\left(X_{\omega_{n}}\right)$ is a singleton which will be denoted by $\pi(\omega)$. Clearly, this defines the coding map

$$
\pi: E_{A}^{\infty} \rightarrow Y
$$

Note that by assuming that $E_{A}^{\infty}$ is equipped with a suitable metric, one immediately verifies, using (a), that $\pi$ is Hölder continuous. The limit set $J_{\mathcal{S}}$ of a pseudo-Markov system $\mathcal{S}$ is then defined by

$$
J_{\mathcal{S}}:=\pi\left(E_{A}^{\infty}\right)
$$

The remainder of this section is devoted to investigations of the geometry, dynamics and topology of this set. In the following a pseudo-Markov system $\mathcal{S}$ is said to be of finite multiplicity if and only if

$$
\left.T:=\sup _{x \in Y}\left\{\operatorname{card}\left\{e \in E: x \in \phi_{e}\left(X_{e}\right)\right)\right\}\right\}<\infty .
$$

The following lemma shows that finite multiplicity is sufficient to ensure that the geometry of the iterations of a pseudo-Markov system is compatible with the geometry arising from the coding. For ease of exposition, in here we use the notation

$$
X_{\omega}:=X_{\omega_{n}} \text { for } \omega=\omega_{1} \ldots \omega_{n} \in E_{A}^{n}, n \in \mathbb{N} .
$$

Lemma 3.2. If $\mathcal{S}$ is a pseudo-Markov system of finite multiplicity, then

$$
J_{\mathcal{S}}=\bigcap_{n \in \mathbb{N}} \bigcup_{\omega \in E_{A}^{n}} \phi_{\omega}\left(X_{\omega}\right) .
$$

Proof. Let $x \in J_{\mathcal{S}}$ be fixed. Then there exists $\omega \in E_{A}^{\infty}$ such that $x=\pi(\omega)$. By definition of $J_{\mathcal{S}}$, it hence follows that $x \in \phi_{\left.\omega\right|_{n}}\left(X_{\omega}\right) \subset \bigcup_{\tau \in E_{A}^{n}} \phi_{\tau}\left(X_{\tau}\right)$, for all $n \in \mathbb{N}$. This implies that

$$
J_{\mathcal{S}} \subset \bigcap_{n \in \mathbb{N}} \bigcup_{\omega \in E_{A}^{n}} \phi_{\omega}\left(X_{\omega}\right) .
$$

(Note that so far we did not use the assumption 'finite multiplicity'). For the opposite inclusion, let $y \in \bigcap_{n=1}^{\infty} \bigcup_{\omega \in E_{A}^{n}} \phi_{\omega}\left(X_{\omega}\right)$, and for each $n \in \mathbb{N}$ consider the set

$$
E_{n}(y):=\left\{\omega \in E_{A}^{n}: y \in \phi_{\omega}\left(X_{\omega}\right)\right\} .
$$

We then have that $\left.\omega\right|_{n} \in E_{n}(y)$, for every $\omega \in E_{n+1}(y)$. This shows that the set $\left\{E_{n}(y): n \in\right.$ $\mathbb{I N}\}$ can be viewed as a tree. Since $\operatorname{card}\left(E_{n}(y)\right) \leq T^{n}<\infty$, Ramsey's Theorem gives that there exists $\omega \in E_{A}^{\infty}$ such that $\left.\omega\right|_{n} \in E_{n}(y)$, for each $n \in \mathbb{N}$. This implies that $\omega \in E_{A}^{\infty}$ and that $y=\pi(\omega)$. 
Finally, we now briefly comment on a topological aspect of the limit set of a pseudo-Markov system $\mathcal{S}$. For this we introduce the 'boundary set'

$$
\Delta(\mathcal{S}):=\left\{\lim _{n \rightarrow \infty} \phi_{e_{n}}\left(x_{n}\right): \lim _{n \rightarrow \infty} e_{n}=\infty \text { for some arbitrary elements } x_{n} \in X_{e_{n}}\right\} .
$$

The following result will be important in the final section where we will use pseudo-Markov systems to study limit sets of infinitely generated Schottky groups.

Proposition 3.3. If $\mathcal{S}$ is a pseudo-Markov system such that $\lim _{e \in E} \operatorname{diam}\left(\phi_{e}\left(X_{e}\right)\right)=0$, then

$$
\overline{J_{\mathcal{S}}}=J_{\mathcal{S}} \cup \bigcup_{\omega \in E_{A}^{*}} \phi_{\omega}\left(\Delta(\mathcal{S}) \cap X_{\omega}\right) .
$$

Proof. Since the matrix $A$ is finitely irreducible, for each $e \in E$ there exists $\omega \in E_{A}^{\infty}$ such that $e \omega \in E_{A}^{\infty}$. Therefore, using the assumption $\lim _{e \in E} \operatorname{diam}\left(\phi_{e}\left(X_{t(e)}\right)\right)=0$, we deduce that $\Delta(\mathcal{S}) \subset \overline{J_{\mathcal{S}}}$. Combining this observation and the continuity of the maps $\phi_{\omega}$ (which implies that $\left.\left.\phi_{\omega}\left(\Delta(\mathcal{S}) \cap X_{\omega}\right) \subset \phi_{\omega}\left(\overline{J_{\mathcal{S}}} \cap X_{\omega}\right) \subset \overline{\phi_{\omega}\left(J_{\mathcal{S}} \cap X_{\omega}\right.}\right) \subset \overline{J_{\mathcal{S}}}\right)$, it follows that

$$
\overline{J_{\mathcal{S}}} \supset J_{\mathcal{S}} \cup \bigcup_{\omega \in E_{A}^{*}} \phi_{\omega}\left(\Delta(\mathcal{S}) \cap X_{\omega}\right) .
$$

In order to prove the reverse inclusion, let $x \in \overline{J_{\mathcal{S}}}$ be fixed. Then there exists a sequence $\left(\omega^{(n)}\right)_{n \in \mathbb{N}}$ of elements $\omega^{(n)} \in E_{A}^{\infty}$ such that $x=\lim _{n \rightarrow \infty} \pi\left(\omega^{(n)}\right)$. For each $k \in \mathbb{N}$, define

$$
E_{k}^{\prime}(x):=\left\{\left.\omega^{(n)}\right|_{k}: n \geq k\right\} \text { and } E_{0}^{\prime}(x):=\emptyset,
$$

and note that if $\tau \in E_{k+1}^{\prime}(x)$ then there exists $\gamma \in E_{k}^{\prime}(x)$ such that $\left.\tau\right|_{k}=\gamma$. This shows that the set $\left\{E_{k}^{\prime}(x): k \in \mathbb{N}\right\}$ can be viewed as a tree rooted at the vertex $E_{0}^{\prime}(x)$. We now distinguish the following two cases. First, suppose that there exists $k \in \mathbb{N}$ such that $E_{k}^{\prime}(x)$ has infinitely many elements, and define

$$
q:=\min \left\{k \geq 0: E_{k}^{\prime}(x) \text { is infinite }\right\} .
$$

Note that $q \geq 1$, and that the set $E_{q-1}^{\prime}(x)$ is finite and non-empty (although it might be equal to the singleton $\{\emptyset\}$ ). Then there exists $\tau \in E_{q-1}^{\prime}(x) \subset E_{A}^{*}$ and an infinite sequence $\left(\omega_{q}^{\left(n_{j}\right)}\right)_{j \in \mathbb{N}}$ of distinct elements of $E$ such that $\tau \omega_{q}^{\left(n_{j}\right)}=\left.\omega^{\left(n_{j}\right)}\right|_{q}$, for all $j \in \mathbb{N}$. By passing to a subsequence if necessary, we may assume without loss of generality that the sequence $\left(\pi\left(\sigma^{q-1}\left(\omega^{\left(n_{j}\right)}\right)\right)\right)_{j \in \mathbb{N}}$ converges to a point $y \in X_{\tau}$. It follows that $y \in \Delta(\mathcal{S})$, and furthermore,

$$
\begin{aligned}
x & =\lim _{j \rightarrow \infty} \pi\left(\omega^{\left(n_{j}\right)}\right)=\lim _{j \rightarrow \infty} \pi\left(\tau\left(\sigma^{q-1}\left(\omega^{\left(n_{j}\right)}\right)\right)\right)=\lim _{j \rightarrow \infty} \phi_{\tau}\left(\pi\left(\sigma^{q-1}\left(\omega^{\left(n_{j}\right)}\right)\right)\right) \\
& =\phi_{\tau}\left(\lim _{j \rightarrow \infty} \pi\left(\sigma^{q-1}\left(\omega^{\left(n_{j}\right)}\right)\right)\right)=\phi_{\tau}(y) \in \phi_{\tau}\left(\Delta(\mathcal{S}) \cap X_{\tau}\right) .
\end{aligned}
$$

This gives the assertion in this first case. Now suppose that the set $E_{k}^{\prime}(x)$ is finite for each $k \in \mathbb{N}$. Since, as mentioned before, these sets form a tree rooted at $E_{0}^{\prime}(x)$, Ramsey's Theorem implies that there exists an infinite path $\omega \in E_{A}^{\infty}$ such that $\left.\omega\right|_{k} \in E_{k}^{\prime}(x)$, for each $k \in$ 
$\mathbb{N}$. Hence, there exists an increasing sequence $\left(n_{k}\right)_{k \in \mathbb{N}}$ such that $\left.\omega\right|_{k}=\left.\omega^{\left(n_{k}\right)}\right|_{k}$, for all $k \in \mathbb{N}$. It follows that $\pi(\omega), \pi\left(\omega^{\left(n_{k}\right)}\right) \in \phi_{\left.\omega\right|_{k}}\left(X_{\omega_{k}}\right)$. Combining this with the fact that $x=\lim _{k \rightarrow \infty} \pi\left(\omega^{\left(n_{k}\right)}\right)$ and $\lim _{k \rightarrow \infty} \operatorname{diam}\left(\phi_{\left.\omega\right|_{k}}\left(X_{\omega_{k}}\right)\right)=0$, we conclude that $x=\pi(\omega) \in J_{\mathcal{S}}$. This completes the proof of the proposition.

\subsection{Summable Families of Functions.}

Definition 3.4. A family $F=\left\{f^{(e)}: X_{e} \rightarrow \mathbb{R} \mid e \in E\right\}$ of continuous functions $f^{(e)}$ is called acceptable if the function

$$
\tilde{F}: \bigcup_{a \in E} \bigcup_{\substack{b \in E \\ A_{a b}=1}}\{a\} \times \phi_{b}\left(X_{b}\right) \rightarrow \mathbb{R}, \text { given by } \tilde{F}(e, x):=f^{(e)}(x),
$$

is uniformly continuous with respect to the metric inherited from the Cartesian product $E \times Y$, where $E$ is assumed to be equipped with the discrete metric.

Since $X_{e}$ is compact for each $e \in E$, it follows that each function $f^{(e)}$ of an acceptable family $F$ is uniformly continuous and of bounded oscilation, where bounded oscilation means that

$$
\sup \left(f^{(e)}\right)-\inf \left(f^{(e)}\right)<+\infty .
$$

Note that the analogous concept of bounded oscilation for graph directed Markov systems in [9] required that in the formula above the supremum over all $e \in E$ is finite. To link the current setting with the previous chapter we now introduce the following special potential function. A function $f: E_{A}^{\infty} \rightarrow \mathbb{R}$ is called amalgamated function induced by $F$ if and only if

$$
f(\omega)=f^{\left(\omega_{1}\right)}(\pi(\sigma(\omega))) \text { for all } \omega=\omega_{1} \omega_{2} \ldots \in E_{A}^{\infty} .
$$

Our convention will be to use lower case letters for the amalgamated function induced by a given summable family of functions. By combining acceptability of the family $F$ and formula (3.1), we immediately obtain the following result.

Proposition 3.5. If a family of funtions $F$ is acceptable, then the amalgamated function $f$ induced by $F$ is finitely acceptable.

Throughout, a family $F=\left\{f^{(e)}: X_{e} \rightarrow \mathbb{R} \mid e \in E\right\}$ is called summable if and only if

$$
\sum_{e \in E} \exp \left(\sup \left(f^{(e)}\right)\right)<\infty
$$

The topological pressure $\mathrm{P}(F)$ of the family $F$ is given by

$$
\mathrm{P}(F):=\lim _{n \rightarrow \infty} \frac{1}{n} \log \sum_{\omega \in E_{A}^{n}} \exp \left(\sup \left(S_{\omega}(F)\right)\right),
$$


where the function $S_{\omega}(F): X_{\omega} \rightarrow \mathbb{R}$ is defined by

$$
S_{\omega}(F):=\sum_{j=1}^{n} f^{\left(\omega_{j}\right)} \circ \phi_{\sigma^{j} \omega} \text { for } \omega=\omega_{1} \omega_{2} \ldots \in E_{A}^{\infty} .
$$

A straightforward calculation shows that if $F$ is summable then

$$
\mathrm{P}(F) \leq \log \sum_{e \in E} \exp \left(\sup \left(f^{(e)}\right)\right)<\infty .
$$

A further link between the symbolic dynamics and pseudo-Markov systems is given by the following.

Proposition 3.6. If $F$ is an acceptable family of functions and $f$ is the amalgamated function induced by $F$, then $\mathrm{P}(F)=\mathrm{P}(f)$.

Proof. First note that for each $\omega=\omega_{1} \ldots \omega_{n} \in E_{A}^{n}$ with $n \in \mathbb{N}$ arbitrary, and for every $\tau=\tau_{1} \tau_{2} \ldots \in E_{A}^{\infty}$ with $A_{\omega_{n} \tau_{1}}=1$, we have

$$
S_{\omega}(F)(\pi(\tau))=\sum_{j=1}^{n} f^{\left(\omega_{j}\right)} \circ \phi_{\sigma^{j} \omega}(\pi(\tau))=\sum_{j=1}^{n} f\left(\sigma^{j-1} \omega \tau\right)=\sum_{j=0}^{n-1} f\left(\sigma^{j} \omega \tau\right)=S_{n} f(\omega \tau)
$$

Hence,

$$
\exp \left(\sup \left(S_{\omega}(F)\right)\right) \geq \exp \left(\sup \left\{S_{n} f(\omega \tau): \tau \in E_{A}^{\infty}, A_{\omega_{n} \tau_{1}}=1\right\}\right)=\exp \left(\sup \left(\left.S_{n} f\right|_{[\omega]}\right)\right) .
$$

This implies that $\mathrm{P}(F) \geq \mathrm{P}(f)$. The opposite inequality is more delicate. Similar as in the proof of Theorem 2.2, one verifies that

$$
\mathrm{P}(F)=\sup \left\{\mathrm{P}_{D}(F): D \subset E \text { finite }\right\} \text {. }
$$

Fix a finite set $D \subset E$. Since for each $e \in D$ the function $f^{(e)}: X_{e} \rightarrow \mathbb{R}$ is uniformly continuous and of finite oscilation, and since $\operatorname{diam}\left(\phi_{\omega}\left(X_{\omega}\right)\right) \leq s^{|\omega|} \operatorname{diam}(Y)$, it follows that for every $\epsilon>0$ there exists a constant $C_{\epsilon}>0$ such that, for all $\omega \in D_{A}^{*}$ and $x, y \in X_{\omega}$,

$$
\exp \left(S_{\omega}(F)(y)\right) \leq C_{\epsilon} e^{\epsilon|\omega|} \exp \left(S_{\omega}(F)(x)\right) .
$$

Now fix $\epsilon>0, \omega=\omega_{1} \ldots \omega_{n} \in E_{A}^{n}$ with $n \in \mathbb{N}$ arbitrary, and some $\tau=\tau_{1} \tau_{2} \ldots \in D_{A}^{\infty}$ with $A_{\omega_{n} \tau_{1}}=1$. Using (3.3) and (3.4), we obtain for each $x \in X_{\omega_{n}}$,

$$
\exp S_{\omega}(F)(x) \leq C_{\epsilon} e^{\epsilon n} \exp \left(S_{\omega}(F)(\pi(\tau))\right)=C_{\epsilon} e^{\epsilon n} \exp \left(S_{n} f(\omega \tau)\right) \leq C_{\epsilon} e^{\epsilon n} \exp \left(\sup \left(\left.S_{n} f\right|_{[\omega]}\right)\right) .
$$

It follows that $\exp \left(\sup \left(S_{\omega}(F)\right)\right) \leq C_{\epsilon} e^{\epsilon n} \exp \left(\sup \left(\left.S_{n} f\right|_{[\omega]}\right)\right)$. Hence, by recalling the definition of the pressure function and by letting $\epsilon$ tend to 0 , we deduce $\mathrm{P}(F) \leq \mathrm{P}(f)$. This completes the proof.

Definition 3.7. With the notation above, a Borel probability measure $m$ on $Y$ is called an $F$-pseudo-conformal measure if $m$ is supported on the limit set $J_{\mathcal{S}}$ and the following two conditions are satisfied. 
(i) For every $\omega \in E_{A}^{*}$ and each Borel set $B \subset X_{\omega}$, we have

$$
m\left(\phi_{\omega}(B)\right)=\int_{B} \exp \left(S_{\omega}(F)-\mathrm{P}(F)|\omega|\right) d m .
$$

(ii) For all incomparable words $\omega, \tau \in E_{A}^{*}$, we have

$$
m\left(\phi_{\omega}\left(X_{\omega}\right) \cap \phi_{\tau}\left(X_{\tau}\right)\right)=0 .
$$

A simple inductive argument shows that for $m$ to be an $F$-pseudo-conformal measure, instead of (3.5) and (3.6) it is enough to require that for every $e \in E$ and for each Borel set $B \subset X_{e}$, we have

$$
m\left(\phi_{e}(B)\right)=\int_{B} \exp \left(f^{(e)}-\mathrm{P}(F)\right) d m,
$$

as well as, for all $a, b \in E$ such that $a \neq b$,

$$
m\left(\phi_{a}\left(X_{a}\right) \cap \phi_{b}\left(X_{b}\right)\right)=0 .
$$

In the following auxiliary result we use the notation

$$
\hat{X}_{a}:=\bigcup_{\substack{b \in E \\ A_{a b}=1}} \phi_{b}\left(X_{b}\right) \subset X_{a} \text { for } a \in E .
$$

Lemma 3.8. With the notation above, let $m$ be a Borel probability measure on the limit set $J_{\mathcal{S}}$ of the pseudo-conformal Markov system $\mathcal{S}$. If $m$ satisfies (3.6) and if (3.5) holds for all Borel sets $A \subset \hat{X}_{e}$ with $e \in E$, then $m$ is an F-pseudo-conformal measure.

Proof. Since $\left(X_{e} \backslash \hat{X}_{e}\right) \cap J_{\mathcal{S}}=\emptyset$ for each $e \in E$, we have that

$$
m\left(X_{e} \backslash \hat{X}_{e}\right)=0 .
$$

Let $x \in \phi_{e}\left(X_{e} \backslash \hat{X}_{e}\right) \cap J_{\mathcal{S}}$ be fixed, for some $e \in E$. Then $x=\pi(\omega)=\phi_{\omega_{1}}(\pi(\sigma(\tau)))$, for some $\omega \in E_{A}^{\infty}$ with $\omega_{1} \neq e$. Hence, it follows that $J_{\mathcal{S}} \cap \phi_{e}\left(X_{e} \backslash \hat{X}_{e}\right) \subset \bigcup_{b \neq e} \phi_{b}\left(X_{b}\right) \cap \phi_{e}\left(X_{e}\right)$, and consequently (3.8) implies

$$
m\left(\phi_{e}\left(X_{e} \backslash \hat{X}_{e}\right)\right)=m\left(J_{\mathcal{S}} \cap \phi_{e}\left(X_{e} \backslash \hat{X}_{e}\right)\right) \leq \sum_{b \neq e} m\left(\phi_{b}\left(X_{b}\right) \cap \phi_{e}\left(X_{e}\right)\right)=0 .
$$

Therefore, using (3.9), we obtain by induction, for each $\omega \in E_{A}^{*}$ and every $B \subset X_{\omega}$,

$$
\begin{aligned}
m\left(\phi_{\omega}(B)\right) & =m\left(\phi_{\omega}\left(B \cap \hat{X}_{\omega}\right)\right)=\int_{B \cap X_{\omega}} \exp \left(S_{\omega}(F)-\mathrm{P}(F)|\omega|\right) d m \\
& =\int_{B} \exp \left(S_{\omega}(F)-\mathrm{P}(F)|\omega|\right) d m .
\end{aligned}
$$


In the following a family of functions $F=\left\{f^{(e)}: X_{e} \rightarrow \mathbb{R} \mid e \in E\right\}$ is called boundedly distorted if and only if there exists a constant $Q>0$ such that for all $e \in E$ and $\omega \in E_{A}^{*}$ with $A_{\omega e}=1$,

$$
\sup \left(\left.S_{\omega}(F)\right|_{\phi_{e}\left(X_{e}\right)}\right)-\inf \left(\left.S_{\omega}(F)\right|_{\phi_{e}\left(X_{e}\right)}\right) \leq Q
$$

Lemma 3.9. If $F$ is a summable and boundedly distorted family of functions, then the amalgamated function induced by $F$ is finitely acceptable and boundedly distorted.

Proof. The assertion is an immediate consequence of (3.3).

A finite word $\omega \tau \in E^{*}$ is called a pseudo-code of an element $x \in Y$ if and only if $\omega, \tau \in E_{A}^{*}$ such that $\phi_{\tau}\left(X_{\tau}\right) \subset X_{\omega}$ and $x \in \phi_{\omega}\left(\phi_{\tau}\left(X_{\tau}\right)\right)$. Note that the word $\omega \tau$ is not required to belong to $E_{A}^{*}$. If in here we do not want to specify the element $x$, we simply say that $\omega \tau$ is a pseudo-code. Similar as for elements of $E_{A}^{*}$, two pseudo-codes are called comparable if one of them is an extension of the other. Also, two pseudo-codes $\omega \tau$ and $\omega \rho$ of an element $x \in Y$ are said to form an essential pair of pseudo-codes of $x$ if $\tau \neq \rho$ and $|\tau|=|\rho|$. Finally, the essential length of the essential pair of pseudo-codes $\omega \tau$ and $\omega \rho$ of $x$ is defined to be equal to $|\omega|$. With these preparations we can now define the notion of a conformal like pseudo-Markov system. Namely, a pseudo-Markov system $S$ is said to be conformal-like if and only if no element of $Y$ admits essential pairs of pseudo-codes of arbitrarily long essential lengths. One easily verifies that a system which satisfies the strong separation condition (that is $\phi_{a}\left(X_{a}\right) \cap \phi_{b}\left(X_{b}\right)=\emptyset$, for all $a, b \in E$ with $a \neq b$ ) is always conformal-like. Also, in the next section we will see that every conformal pseudo-Markov systems is conformal-like. Now, the following theorem gives the main result concerning conformal-like systems and summable families of functions.

Theorem 3.10. Let $\mathcal{S}=\left\{\phi_{e}: e \in E\right\}$ be a conformal-like pseudo-Markov system. If $F$ is a summable and boundedly distorted family of functions, then there exists an F-pseudoconformal measure $m_{F}$ on $J_{\mathcal{S}}$. Moreover, we have that $m_{F}=\tilde{m}_{F} \circ \pi^{-1}$, where $\tilde{m}_{F}$ is the $f$-pseudo-conformal measure whose existence follows from Theorem 2.6 and Lemma 3.9.

Proof. Let $m_{F}=\tilde{m}_{F} \circ \pi^{-1}$. In order to show that $m_{F}$ is an $F$-pseudo-conformal measure, assume by way of contradiction that $m_{F}\left(\phi_{\rho}\left(X_{\rho}\right) \cap \phi_{\tau}\left(X_{\tau}\right)\right)>0$, for two incomparable words $\rho, \tau \in E_{A}^{*}$. Without loss of generality we can assume that $\rho$ and $\tau$ are of the same length, say $q \in \mathbb{N}$. Then define, for $n \in \mathbb{N}$,

$$
\left.V:=\phi_{\rho}\left(X_{\rho}\right) \cap \phi_{\tau}\left(X_{\tau}\right)\right) \text { and } V_{n}:=\bigcup_{\omega \in E_{A}^{n}} \phi_{\omega}\left(X_{\omega} \cap V\right) .
$$

Since each element of $V_{n}$ admits at least one essential pair of pseudo-codes of essential length equal to $n$, and since the system $\mathcal{S}$ is confomal-like, it follows that

$$
\bigcap_{k=1}^{\infty} \bigcup_{n=k}^{\infty} V_{n}=\emptyset \text {. }
$$


On the other hand, we have $V_{n} \supset \pi\left(\sigma^{-n}\left(\pi^{-1}(V)\right)\right)$, which implies that $\pi^{-1}\left(V_{n}\right) \supset \sigma^{-n}\left(\pi^{-1}(V)\right)$. By Theorem 2.10 we know that there exists a $\sigma$-invariant Borel probability measure $\tilde{\mu}_{F}$ equivalent to $\tilde{m}_{F}$. Hence, $\tilde{\mu}_{F}\left(\pi^{-1}\left(V_{n}\right)\right) \geq \tilde{\mu}_{F}\left(\sigma^{-n}\left(\pi^{-1}(V)\right)\right)=\tilde{\mu}_{F} \circ \pi^{-1}(V)>0$, and therefore,

$$
\tilde{\mu}_{F} \circ \pi^{-1}\left(\bigcap_{k=1}^{\infty} \bigcup_{n=k}^{\infty} V_{n}\right) \geq \tilde{\mu}_{F} \circ \pi^{-1}(V)>0 .
$$

In particular, this gives that $\bigcap_{k=1}^{\infty} \bigcup_{n=k}^{\infty} V_{n} \neq \emptyset$, and hence we obtain a contradiction. This shows that property (3.6) is satisfied. In order to prove (3.5), fix $\omega=\omega_{1} \ldots \omega_{n} \in E_{A}^{n}$ as well as a Borel set $B \subset X_{\omega}$, and define

$$
\pi_{\omega}^{-1}(B):=\left\{\tau \in \pi^{-1}(B): A_{\omega_{n} \tau_{1}}=1\right\} .
$$

Consider some element $\tau=\tau_{1} \tau_{2} \ldots \in \pi^{-1}(B) \backslash \pi_{\omega}^{-1}(B)$. We then have $A_{\omega_{n} \tau_{1}}=0$ and $\pi(\tau) \in B \subset X_{\omega}$. This implies that $\pi(\tau)=\phi_{e}\left(X_{e}\right)$, for some $e \in E$ with $A_{\omega_{n} e}=1$. Hence, $e \neq \tau_{1}$ and $\pi(\tau) \in \phi_{e}\left(X_{e}\right) \cap \phi_{\tau_{1}}\left(X_{\tau_{1}}\right)$, and therefore,

$$
\pi\left(\pi^{-1}(B) \backslash \pi_{\omega}^{-1}(B)\right) \subset \bigcup_{e \neq b} \phi_{e}\left(X_{e}\right) \cap \phi_{b}\left(X_{b}\right) .
$$

By combining this inclusion with (3.6), we obtain

$$
\begin{aligned}
\tilde{m}_{F}\left(\pi^{-1}(B) \backslash \pi_{\omega}^{-1}(B)\right) & \leq \tilde{m}_{F} \circ \pi^{-1}\left(\bigcup_{e \neq b} \phi_{e}\left(X_{e}\right) \cap \phi_{b}\left(X_{b}\right)\right) \\
& =m_{F}\left(\bigcup_{e \neq b} \phi_{e}\left(X_{e}\right) \cap \phi_{b}\left(X_{b}\right)\right)=0 .
\end{aligned}
$$

Note that one immediately verifies that

$$
\pi^{-1}\left(\phi_{\omega}(B)\right) \supset \sigma_{\omega}^{-n} \circ \pi_{\omega}^{-1}(B) .
$$

Now, consider some element $\rho \in \pi^{-1}\left(\phi_{\omega}(B)\right) \backslash\left(\sigma_{\omega}^{-n} \circ \pi_{\omega}^{-1}(B)\right)$. Then $\pi(\rho)=\phi_{\omega e}(x)$, where $A_{\omega_{n} e}=1, \phi_{e}(x) \in B$ and $\rho \notin \sigma_{\omega}^{-n} \circ \pi_{\omega}^{-1}(B)$. If $\left.\rho\right|_{n+1}=\omega e$ then $x=\pi\left(\sigma^{n+1} \rho\right)$, and it follows that $\pi\left(e \sigma^{n+1} \rho\right)=\phi_{e}\left(\pi\left(\sigma^{n+1} \rho\right)\right)=\phi_{e}(x)$. Consequently, $\rho=\omega e \sigma^{n+1}(\rho) \in \sigma_{\omega}^{-n} \circ \pi_{\omega}^{-1}(B)$, and hence $\left.\rho\right|_{n+1} \neq \omega i$. This shows that

$$
\pi^{-1}\left(\phi_{\omega}(B)\right) \backslash\left(\sigma_{\omega}^{-n} \circ \pi_{\omega}^{-1}(B)\right) \subset \pi^{-1}\left(\bigcup_{\substack{\tau, \eta \in E_{A}^{n+1} \\ \tau \neq \eta}} \phi_{\tau}\left(X_{\tau}\right) \cap \phi_{\eta}\left(X_{\eta}\right)\right) .
$$

Using (3.6) and the definition of the measure $m_{F}$, it follows that

$$
\tilde{m}_{F}\left(\pi^{-1}\left(\phi_{\omega}(B)\right) \backslash \sigma_{\omega}^{-n} \circ \pi_{\omega}^{-1}(B)\right)=0 .
$$


Eventually, by combining the latter equality, (3.12) and (2.5), we obtain

$$
\begin{aligned}
m_{F}\left(\phi_{\omega}(B)\right) & =\tilde{m}_{F} \circ \pi^{-1}\left(\phi_{\omega}(B)\right)=\tilde{m}_{F}\left(\sigma_{\omega}^{-n} \circ \pi_{\omega}^{-1}(B)\right) \\
& =\int_{\pi_{\omega}^{-1}(B)} \exp \left(S_{n} f(\omega \rho)-\mathrm{P}(f) n\right) d \tilde{m}_{F}(\rho) \\
& =\int_{\pi_{\omega}^{-1}(B)} \exp \left(S_{\omega} F(\pi(\rho))-\mathrm{P}(F) n\right) d \tilde{m}_{F}(\rho) \\
& =\int_{\pi^{-1}(B)} \exp \left(S_{\omega} F(\pi(\rho))-\mathrm{P}(F) n\right) d \tilde{m}_{F}(\rho) \\
& =\int_{B} \exp \left(S_{\omega} F(x)-\mathrm{P}(F) n\right) d \tilde{m} \circ \pi^{-1}(x)=\int_{B} \exp \left(S_{\omega} F-\mathrm{P}(F) n\right) d m_{F} .
\end{aligned}
$$

This shows that (3.5) is satisfied, and hence an application of Lemma 3.8 then completes the proof of the theorem.

\section{Conformal Pseudo-Markov Systems}

\subsection{Preliminaries for Conformal Pseudo-Markov Systems.}

In this section we consider a particular class of pseudo-Markov systems which we call conformal pseudo-Markov systems. These systems are represented in some Euclidean space $\mathbb{R}^{d}$, and this allows to study the fractal and topological structure of their limit sets. Before giving the definition of a conformal pseudo-Markov system, recall that a set $X \subset \mathbb{R}^{d}$ is called $K$-quasiconvex, for some $K \geq 1$, if for every pair of points $x, y \in X$ there exists a piecewise smooth path in $X$ joining $x$ and $y$ of length less than or equal to $K|x-y|$.

Definition 4.1. Let $\mathcal{S}=\left\{\phi_{e}: X_{e} \rightarrow Y \mid e \in E\right\}$ be a pseudo-Markov system. Then $\mathcal{S}$ is called a conformal pseudo-Markov system if and only if $Y$ is a compact subset of the Euclidean space $\mathbb{R}^{d}$, for some $d \in \mathbb{N}$, and if the following conditions are satisfied.

(1) (Quasi-Convexity) There exists a constant $K \geq 1$ such that $X_{e}$ and $\phi_{e}\left(X_{e}\right)$ are $K$ quasi-convex, for all $e \in E$.

(2) (Derivative Decay) $\lim _{e \in E}\left\|\phi_{e}^{\prime}\right\|=0$.

(3) (Cone Condition) There exists $\theta \in(0, \pi / 2)$ such that for all $e \in E$ and $x \in X_{e}$, there exists an open cone $\operatorname{Cone}(x, \theta)$ with vertex $x$ and central angle $\theta$ such that $\operatorname{Cone}(x, \theta) \subset$ $\operatorname{Int}\left(\phi_{e}\left(X_{e}\right)\right)$, the interior being taken with respect to the Euclidean topology of $\mathbb{R}^{d}$.

(4) (Conformal Extension) For each $e \in E$, there exists an open connected set $V_{e} \subset Y$ such that $X_{e} \subset V_{e}$, and such that the map $\phi_{e}: X_{e} \rightarrow Y$ extends to a $C^{1+\epsilon}$-conformal diffeomorphism from $V_{e}$ to $Y$.

(5) (Pre-Distortion) There exist $L \geq 1$ and $\alpha>0$ such that for all ab $\in E_{A}^{2}$ and all $x, y \in \phi_{b}\left(X_{b}\right)$,

$$
|| \phi_{a}^{\prime}(y)|-| \phi_{a}^{\prime}(x)|| \leq L \frac{|y-x|^{\alpha}}{\left\|\left(\left.\phi_{a}^{\prime}\right|_{\phi_{b}\left(X_{b}\right)}\right)^{-1}\right\|} .
$$


Let us first collect a few geometric consequences which arise from the Pre-Distortion condition.

Lemma 4.2. If $\mathcal{S}=\left\{\phi_{e}: X_{e} \rightarrow Y \mid e \in E\right\}$ is a conformal pseudo-Markov system, then for all $\omega \in E_{A}^{*}$ and $b \in E$ such that $\omega b \in E_{A}^{*}$,

$$
|\log | \phi_{\omega}^{\prime}(y)|-\log | \phi_{\omega}^{\prime}(x)|| \leq L\left(1-s^{\alpha}\right)^{-1}|y-x|^{\alpha}, \text { for all } x, y \in \phi_{b}\left(X_{b}\right) .
$$

Proof. Let $n:=|\omega|$, and define $z_{0}:=z$ and $z_{k}:=\phi_{\omega_{n-k+1}} \circ \phi_{\omega_{n-k+2}} \circ \cdots \circ \phi_{\omega_{n}}(z)$, for arbitrary $z \in \phi_{b}\left(X_{b}\right)$ and for all $1 \leq k \leq n$. Using the Pre-Distortion condition (5) in Definition 4.1, it follows for $x, y \in \phi_{b}\left(X_{b}\right)$,

$$
\begin{aligned}
\left|\log \left(\left|\phi_{\omega}^{\prime}(y)\right|\right)-\log \left(\left|\phi_{\omega}^{\prime}(x)\right|\right)\right| & =\left|\sum_{j=1}^{n} \log \left(1+\frac{\left|\phi_{\omega_{j}}^{\prime}\left(y_{n-j}\right)\right|-\left|\phi_{\omega_{j}}^{\prime}\left(x_{n-j}\right)\right|}{\left|\phi_{\omega_{j}}^{\prime}\left(x_{n-j}\right)\right|}\right)\right| \\
& \leq \sum_{j=1}^{n} \|\left(\phi_{\omega_{j}}^{\prime}\right)^{-1}||\left|\phi_{\omega_{j}}^{\prime}\left(y_{n-j}\right)\right|-\left|\phi_{\omega_{j}}^{\prime}\left(x_{n-j}\right)\right| \mid \\
& \leq \sum_{j=1}^{n} L\left|y_{n-j}-x_{n-j}\right|^{\alpha} \\
& \leq L \sum_{j=1}^{n} s^{\alpha(n-j)}|y-x|^{\alpha} \leq L\left(1-s^{\alpha}\right)^{-1}|y-x|^{\alpha} .
\end{aligned}
$$

Let us define

$$
Q:=L\left(1-s^{\alpha}\right)^{-1}(\operatorname{diam}(Y))^{\alpha} .
$$

An immediate consequence of the previous lemma is that each conformal pseudo-Markov system has the following property.

Bounded Distortion Property. For all $\omega \in E_{A}^{*}$ and $b \in E$ such that $\omega b \in E_{A}^{*}$, we have for all $x, y \in \phi_{b}\left(X_{b}\right)$,

$$
e^{-Q} \leq \frac{\left|\phi_{\omega}^{\prime}(y)\right|}{\left|\phi_{\omega}^{\prime}(x)\right|} \leq e^{Q}
$$

The following lemma gives a sufficient condition for the Pre-Distortion property to hold.

Lemma 4.3. Suppose that $\mathcal{S}=\left\{\phi_{e}: X_{e} \rightarrow Y \mid e \in E\right\}$ is a pseudo-Markov system, with $d \geq 2$, satisfying conditions (1)-(4) in Definition 4.1, and suppose that there exists $\kappa>0$ such that

$$
\operatorname{dist}\left(\phi_{b}\left(X_{b}\right), \mathbb{R}^{d} \backslash X_{a}\right) \geq \kappa \operatorname{diam}\left(\phi_{b}\left(X_{b}\right)\right) \text { for all } a b \in E_{A}^{2} \text {. }
$$

Then $\mathcal{S}$ satisfies the Pre-Distortion property (5), and hence $\mathcal{S}$ is a conformal pseudo-Markov system. 
Proof. The assertion follows immediately from Theorem 4.1.2 and Theorem 4.1.3 in [9].

Proposition 4.4. If $\mathcal{S}$ is a conformal pseudo-Markov system, then

$$
\overline{J_{\mathcal{S}}}=J_{\mathcal{S}} \cup \bigcup_{\omega \in E_{A}^{*}} \phi_{\omega}\left(\Delta(\mathcal{S}) \cap X_{\omega}\right) .
$$

Proof. Using the Mean Value Inequality, conditions (1) and (2) in Definition 4.1, and the assumption that $Y$ is bounded, we immediately obtain that

$$
\lim _{e \in E} \operatorname{diam}\left(\phi_{e}\left(X_{e}\right)\right)=0 .
$$

Hence, we can apply Proposition 3.3, which then gives the assertion.

Lemma 4.5. There exists $\beta>0$ such that $\phi_{\omega}\left(\phi_{\tau}\left(X_{\tau}\right)\right)$ contains an open central cone with vertex $\phi_{\omega}\left(\phi_{\tau}(x)\right)$ and angle measure $\beta$, for each $x \in Y$ and every pseudo-code $\omega \tau$ of $x$.

Proof. By using the Cone Condition (3) in Definition 4.1, the proof is exactly the same as the proof of formula (4.14) in [9], and will therefore be omitted.

One easily verifies that $\phi_{\omega} \circ \phi_{\rho}\left(\operatorname{Int}\left(X_{\rho}\right)\right) \cap \phi_{\tau} \circ \phi_{\gamma}\left(\operatorname{Int}\left(X_{\gamma}\right)\right)=\emptyset$, for any two incomparable pseudo-codes $\omega \rho$ and $\tau \gamma$. Hence, as an immediate consequence of the previous lemma we obtain the following corollary. In here $\lambda_{d-1}$ refers to the $(d-1)$-dimensional Lebesgue on the unit sphere $\mathbb{S}^{d-1} \subset \mathbb{R}^{d}$.

Corollary 4.6. With $\beta$ as in the previous lemma, we have that for each $x \in Y$ there are at most $\lambda_{d-1}\left(\mathbb{S}^{d-1}\right) / \beta$ mutually incomparable pseudo-codes of $x$.

Lemma 4.7. Every conformal pseudo-Markov system $\mathcal{S}$ is a conformal-like system.

Proof. Suppose on the contrary that there exists a point $x \in Y$ with the following properties. There exists an infinite increasing sequence $\left(n_{k}\right)_{k \in \mathbb{N}}$ of positive integers, and there exist words $\omega^{(k)}, \tau^{(k)}, \rho^{(k)} \in E_{A}^{*}$, for each $k \in \mathbb{N}$, such that $\phi_{\tau^{(k)}}\left(X_{\tau^{(k)}}\right) \cup \phi_{\rho^{(k)}}\left(X_{\tau^{(k)}}\right) \subset X_{\omega^{(k)}}$, such that the words $\tau^{(k)}$ and $\rho^{(k)}$ are incomparable, and

$$
\lim _{k \rightarrow \infty}\left|\omega^{(k)}\right|=\infty
$$

as well as

$$
x \in \phi_{\omega^{(k)}} \circ \phi_{\tau^{(k)}}\left(X_{\tau^{(k)}}\right) \cap \phi_{\omega^{(k)}} \circ \phi_{\rho^{(k)}}\left(X_{\rho^{(k)}}\right) \text { for all } k \in \mathbb{N} .
$$

We now construct by induction for each $n \in \mathbb{N}$ a set $C_{n}$ which contains at least $n+1$ mutually incomparable pseudo-codes of $x$. The existence of such a set for large $n$ will then clearly contradict the statement in Corollary 4.6, and hence will finish the proof. Define

$$
C_{1}:=\left\{\omega^{(1)} \tau^{(1)}, \omega^{(1)} \rho^{(1)}\right\},
$$


and suppose that the set $C_{n}$ has been obtained, for some $n \in \mathbb{N}$. In view of (4.4), there exists $k_{n} \in \mathbb{N}$ such that

$$
\left|\omega^{\left(k_{n}\right)}\right|>\max \left\{|\xi|: \xi \in C_{n}\right\} .
$$

If $\omega^{\left(k_{n}\right)} \rho^{\left(k_{n}\right)}$ does not extend any word from $C_{n}$, then we have by $(4.5)$ that $\omega^{\left(k_{n}\right)} \rho^{\left(k_{n}\right)}$ is not comparable with any element of $C_{n}$. We then obtain $C_{n+1}$ from $C_{n}$ by adding the word $\omega^{\left(k_{n}\right)} \rho^{\left(k_{n}\right)}$ to $C_{n}$. Similarly, if $\omega^{\left(k_{n}\right)} \tau^{\left(k_{n}\right)}$ does not extend any word from $C_{n}$, then form $C_{n+1}$ by adding $\omega^{\left(k_{n}\right)} \tau^{\left(k_{n}\right)}$ to $C_{n}$. On the other hand, if $\omega^{\left(k_{n}\right)} \rho^{\left(k_{n}\right)}$ extends an element $\alpha \in C_{n}$ and $\omega^{\left(k_{n}\right)} \tau^{\left(k_{n}\right)}$ extends an element $\beta \in C_{n}$, then we obtain from (4.5) that $\alpha=\left.\omega^{\left(k_{n}\right)}\right|_{|\alpha|}$ and $\beta=\left.\omega^{\left(k_{n}\right)}\right|_{|\beta|}$. Since $C_{n}$ consists of mutually incomparable words, this implies that $\alpha=\beta$. Now, form $C_{n+1}$ by removing $\alpha=\beta$ from $C_{n}$ and then adding both $\omega^{\left(k_{n}\right)} \rho^{\left(k_{n}\right)}$ and $\omega^{\left(k_{n}\right)} \tau^{\left(k_{n}\right)}$. Note that no element $\gamma \in C_{n} \backslash\{\alpha\}$ is comparable with $\omega^{\left(k_{n}\right)} \rho^{\left(k_{n}\right)}$ or $\omega^{\left(k_{n}\right)} \tau^{\left(k_{n}\right)}$, since otherwise $\gamma=\left.\omega^{\left(k_{n}\right)}\right|_{|\gamma|}$, and consequently, $\gamma$ would be comparable with $\alpha$. Since also $\omega^{\left(k_{n}\right)} \rho^{\left(k_{n}\right)}$ and $\omega^{\left(k_{n}\right)} \tau^{\left(k_{n}\right)}$ are not comparable, it follows that $C_{n+1}$ consists of mutually incomparable pseudocodes of $x$. This completes our inductive construction, and hence finishes the proof.

\subsection{Pressure Functions, Conformal Measures and Bowen's Formula.}

Throughout this section let $\mathcal{S}=\left\{\phi_{e}: X_{e} \rightarrow Y: e \in E\right\}$ be a given conformal pseudo-Markov system. For each $t \geq 0$, consider the following family of functions

$$
t \log :=\left\{t \log \left|\phi_{e}^{\prime}\right|: e \in E\right\} .
$$

Lemma 4.8. $t \log$ is an acceptable and boundedly distorted family of functions, for each $t \geq 0$.

Proof. The assertion is an immediate consequence of Lemma 4.2.

Let $\zeta: E_{A}^{\infty} \rightarrow \mathbb{R}$ be the amalgamated function induced by the family Log, that is for every $\omega=\omega_{1} \omega_{2} \ldots \in E_{A}^{\infty}$ we have

$$
\zeta(\omega):=\log \left|\phi_{\omega_{1}}^{\prime}(\pi(\sigma \omega))\right|
$$

Then $t \zeta$ is the amalgamated function induced by the family $t \log$, for each $t \geq 0$. Hence, by combining Proposition 3.6 and Lemma 4.8, we have

$$
\mathrm{P}(t \zeta)=\mathrm{P}(t \log )
$$

and in the sequel we will simply write $\mathrm{P}(t)$ to denote their common value. For the following theorem note that by condition (2) (Derivative Decay) in Definition 4.1, we have that there exists a unique number $\theta(\mathcal{S}) \in[0, \infty)$ such that the family $t \log$ is summable for all $t>\theta(\mathcal{S})$, and not summable for all $t<\theta(\mathcal{S})$. Clearly, $\mathrm{P}(\theta(\mathcal{S}))<\infty$ if and only if $\theta(\mathcal{S}) \log$ is summable. Finally, recall that a Borel probability measure $m_{t}$ on $J_{\mathcal{S}}$ is a $t$ Log-pseudo-conformal measure if and only if

$$
m_{t}\left(\phi_{\omega}\left(X_{\omega}\right) \cap \phi_{\tau}\left(X_{\tau}\right)\right)=0 \text { for all incomparable words } \omega, \tau \in E_{A}^{*},
$$


and

$$
m_{t}\left(\phi_{\omega}(B)\right)=\int_{B} e^{-\mathrm{P}(t)}\left|\phi_{\omega}^{\prime}\right|^{t} d m_{t} \text { for all } \omega \in E_{A}^{*} \text {, and } B \subset X_{\omega} \text { Borel. }
$$

If $\mathrm{P}(t)=0$, then the measure $m_{t}$ is called $t$-conformal.

Theorem 4.9. If $t \log$ is a summable family, and hence in particular if $t>\theta(S)$, then there exists a $t$ Log-pseudo-conformal measure $m_{t}$ on $J_{\mathcal{S}}$.

Proof. The assertion follows immediately by combining Theorem 3.10, Lemma 4.7 and Lemma 4.8.

The following proposition collects some of the basic properties of the pressure function $t \mapsto$ $\mathrm{P}(t)$. These properties are immediate consequences of the Hölder inequality and the fact that $\left\|\phi_{e}^{\prime}\right\| \leq s<1$ for all $e \in E$, and we omit the proof.

Proposition 4.10. For the pressure function $\mathrm{P}:[0, \infty) \rightarrow \mathbb{R}$ of a conformal pseudo-Markov system $\mathcal{S}$, the following holds.

(a) $\mathrm{P}$ is decreasing.

(b) $\left.\mathrm{P}\right|_{[\theta(\mathcal{S}), \infty)}$ is strictly decreasing.

(c) $\lim _{t \rightarrow \infty} \mathrm{P}(t)=-\infty$.

(d) $\left.\mathrm{P}\right|_{[\theta(\mathcal{S}), \infty)}$ is convex, and hence, continuous.

For ease of exposition, let us introduce the following two parameters.

$$
h=h(\mathcal{S}):=\operatorname{HD}\left(J_{\mathcal{S}}\right)
$$

and

$$
h^{*}=h^{*}(\mathcal{S}):=\sup \left\{\operatorname{HD}\left(J_{\left.\mathcal{S}_{D}\right)}\right): D \subset E \text { finite }\right\}
$$

where $\mathcal{S}_{D}:=\left\{\phi_{e}: e \in D\right\}$. Also, we introduce the concept of thinness, which will turn out to be crucial in the following.

Definition 4.11. A conformal pseudo-Markov system $\mathcal{S}$ is called thin if and only if

$$
\theta(\mathcal{S})<h^{*}(\mathcal{S})
$$

We remark that an equivalent formulation of thinnes is that there exists a positive constant $\kappa=\kappa(\mathcal{S})<h^{*}(\mathcal{S})$ such that

$$
\sum_{e \in E}\left\|\phi_{e}^{\prime}\right\|^{\kappa}<\infty
$$

Note that thinness implies in particular that

$$
\sum_{e \in E}\left\|\phi_{e}^{\prime}\right\|^{h^{*}}<\infty
$$

Furthermore, if for instance $\theta(\mathcal{S})=0$, then the underlying pseudo-Markov system is thin. The following auxiliary result will turn out to be useful in the proof of the next theorem. 
Lemma 4.12. If $\mathcal{S}$ is a finite (irreducible) conformal pseudo-Markov system, then $\mathrm{P}(h(\mathcal{S}))=$ 0 .

Proof. Since $0 \leq \mathrm{P}(0)<\infty$, Proposition 4.10 implies that there exists a unique $\eta \geq 0$ such that $\mathrm{P}(\eta)=0$. Let $m_{\eta}$ be the associated $\eta$-conformal measure (see Theorem 4.9). Note that the system $\mathcal{S}^{\prime}=\left\{\phi_{a}: \phi_{b}\left(X_{b}\right) \rightarrow \phi_{a}\left(X_{a}\right) \mid a, b \in E, A_{a b}=1\right\}$ satisfies all the requirements of a graph directed Markov system (see [9], page 71-72). Also note that the limit sets of $\mathcal{S}^{\prime}$ and $\mathcal{S}$ coincide, and that the measure $m_{\eta}$ is an $\eta$-conformal measure also for $\mathcal{S}^{\prime}$. The proof now follows from Theorem 4.2.11 in [9], where primitivity can easily be replaced by irreducibility.

The following theorem represents the main result of this section. The theorem gives a extension of Bowen's formula [2] to the setting of thin conformal pseudo-Markov systems.

Theorem 4.13. If $\mathcal{S}$ is a thin conformal pseudo-Markov system, then $h(\mathcal{S})$ is the unique zero of the pressure function $\mathrm{P}$, and $h(\mathcal{S})=h^{*}(\mathcal{S})$.

Proof. Put $h:=h(\mathcal{S}), h^{*}:=h^{*}(\mathcal{S})$, and

$$
u:=\inf \{s \geq 0: \mathrm{P}(s) \leq 0\} .
$$

Our first aim is to show that $h \leq u$. For this, fix $t>u$ and observe that Proposition 4.10 implies that $\mathrm{P}(t)<0$. Since $X_{e}$ is $K$-quasi-convex for all $e \in E$ (see Definition 4.1 (1)), we have by definition of $\mathrm{P}$, for all $n \in \mathbb{N}$ sufficiently large,

$$
\begin{aligned}
\sum_{\omega \in E_{A}^{n}}\left(\operatorname{diam}\left(\phi_{\omega}\left(X_{\omega}\right)\right)\right)^{t} & \leq K^{t} \sum_{\omega \in E_{A}^{n}}\left\|\phi_{\omega}^{\prime}\right\|^{t}\left(\operatorname{diam}\left(X_{\omega}\right)\right)^{t} \\
& \leq K^{t}(\operatorname{diam}(Y))^{t} \sum_{\omega \in E_{A}^{n}}\left\|\phi_{\omega}^{\prime}\right\|^{t} \leq K^{t}(\operatorname{diam}(Y))^{t} \exp \left(\frac{1}{2} \mathrm{P}(t) n\right) .
\end{aligned}
$$

Letting $n$ tend to infinity and using the fact that by Lemma 3.2 we have that $\left\{\phi_{\omega}\left(X_{\omega}\right): \omega \in\right.$ $\left.E_{A}^{n}\right\}$ is a covering of $J_{\mathcal{S}}$, we conclude that the $t$-dimensional Hausdorff measure of $J_{\mathcal{S}}$ vanishes. This shows that $h \leq t$, and hence by letting $t$ tend to $u$ from above, it follows that

$$
h \leq u \text {. }
$$

Our next aim is to show that $u \leq h^{*}$. For ease of exposition and without loss of generality, let us assume that $E=\mathbb{N}$. Since $A$ is finitely irreducible, there exists $p \in \mathbb{N}$ sufficiently large such that $\Xi \subset\{1,2, \ldots, p\}^{*}$. For $k \geq p$, let

$$
\mathcal{S}_{k}:=\mathcal{S}_{\{1, \ldots, k\}}
$$

and let $h_{k}:=h\left(S_{k}\right)$. One immediately verifies that $\lim _{k \rightarrow \infty} h_{k}=h$. Note that by Lemma 4.12 and Theorem 4.9, for every $k \geq p$ there exists an $h_{k}$-conformal measure associated with $\mathcal{S}_{k}$. Also, since the system $\mathcal{S}$ is thin, there exists $0<\kappa<h^{*}$ and $q \geq p$ such that

$$
\sum_{j=q+1}^{\infty}\left\|\phi_{e}^{\prime}\right\|^{\kappa}<\frac{1}{2} .
$$


Finally, by choosing $p$ sufficiently large, we can always assume without loss of generality that $p \geq \min \left\{m: h_{m} \geq \kappa\right\}$. With these preparations we now obtain for $k \geq q$, using the $h_{k}$-conformality of the measure $m_{k}$,

$$
\begin{aligned}
1 & =\sum_{j=1}^{k} m_{k}\left(\phi_{j}\left(X_{j}\right)\right)=\sum_{j=1}^{q} m_{k}\left(\phi_{j}\left(X_{j}\right)\right)+\sum_{j=q+1}^{k} m_{k}\left(\phi_{j}\left(X_{j}\right)\right) \\
& \leq \sum_{j=1}^{q} m_{k}\left(\phi_{j}\left(X_{j}\right)\right)+\sum_{j=q+1}^{k}\left\|\phi_{j}^{\prime}\right\|^{h_{k}} m_{k}\left(X_{j}\right) \\
& \leq \sum_{j=1}^{q} m_{k}\left(\phi_{j}\left(X_{j}\right)\right)+\sum_{j=q+1}^{k}\left\|\phi_{j}^{\prime}\right\|^{\kappa} \leq \frac{1}{2}+\sum_{j=1}^{q} m_{k}\left(\phi_{j}\left(X_{j}\right)\right) .
\end{aligned}
$$

This implies that $\sum_{j=1}^{q} m_{k}\left(\phi_{j}\left(X_{j}\right)\right) \geq 1 / 2$, and hence there exists $a \in\{1,2, \ldots, q\}$ such that

$$
m_{k}\left(\phi_{a}\left(X_{a}\right)\right) \geq \frac{1}{2 q}
$$

Since $k \geq q \geq p$, for each $e \in\{1,2, \ldots, k\}$ there exists $\rho \in \Xi$ such that e $\rho a$ is an admissable word in $\mathcal{S}_{k}$. Therefore, using (4.2) and (4.11), we obtain

$$
m_{k} \phi_{e}\left(X_{e}\right) \geq m_{k}\left(\phi_{e \rho}\left(\phi_{a}\left(X_{a}\right)\right)\right) \geq e^{-Q h_{k}}\left\|\phi_{e \rho}^{\prime}\right\|^{h_{k}} m_{k}\left(\phi_{a}\left(X_{a}\right)\right) \geq(2 q)^{-1} e^{-Q h_{k}}\left\|\phi_{e \rho}^{\prime}\right\|^{d} .
$$

Since for every $e \in E$ there are at most $\# \Xi$ possible paths of the form $e \rho$, we deduce that $\inf \left\{m_{k}\left(\phi_{e}\left(X_{e}\right)\right): k \geq q\right\}>0$. Consequently,

$$
T_{q}:=\min \left\{m_{k}\left(\phi_{e}\left(X_{e}\right)\right): 1 \leq e \leq q, k \geq q\right\}>0
$$

For $n \in \mathbb{N} \cup\{\infty\}$ and $k \geq q$, let $E_{k}^{n}$ denote the set of all $A$-admissible words of length $n$ over the alphabet $\{1,2, \ldots, k\}$. For all $t \geq 0$, set

$$
Z_{n}(t, k):=\sum_{\omega \in E_{k}^{n}}\left\|\phi_{\omega}^{\prime}\right\|^{t} \text { and } \Sigma_{t}:=Z_{1}(t, \infty)
$$


Using the $h_{k}$-conformality of $m_{k}$ as well as (4.2) and the definition of $T_{q}$, we now obtain for all $n \in \mathbb{N}$ and $k \geq q$,

$$
\begin{aligned}
& Z_{n+1}\left(h^{*}, k\right)=\sum_{\omega \in E_{k}^{n}} \sum_{\substack{e \leq q \\
A_{\omega_{n} e=1}}}\left\|\phi_{\omega e}^{\prime}\right\|^{h^{*}}+\sum_{\omega \in E_{k}^{n}} \sum_{\substack{e>q \\
A_{\omega_{n} e=1}}}\left\|\phi_{\omega e}^{\prime}\right\|^{h^{*}} \\
& \leq \sum_{\omega \in E_{k}^{n}} \sum_{\substack{e \leq q \\
A_{\omega_{n} e=1}}}\left\|\phi_{\omega}^{\prime}\right\|^{h_{k}}\left\|\phi_{e}^{\prime}\right\|^{h^{*}}+\sum_{\omega \in E_{k}^{n}} \sum_{e>q}\left\|\phi_{\omega}^{\prime}\right\|^{h^{*}}\left\|\phi_{e}^{\prime}\right\|^{h^{*}} \\
& \leq e^{Q h_{k}} \sum_{\omega \in E_{k}^{n}} \sum_{\substack{e \leq q \\
A_{\omega_{n} e=1}}}\left\|\phi_{e}^{\prime}\right\|^{h^{*}} \frac{m_{k}\left(\phi_{\omega}\left(\phi_{e}\left(X_{e}\right)\right)\right)}{m_{k}\left(\phi_{e}\left(X_{e}\right)\right)}+\sum_{\omega \in E_{k}^{n}}\left\|\phi_{\omega}^{\prime}\right\|^{h^{*}} \sum_{e>q}\left\|\phi_{e}^{\prime}\right\|^{h_{k}} \\
& \leq T_{q}^{-1} e^{Q h_{k}} \sum_{\omega \in E_{k}^{n}} \sum_{\substack{e \leq q \\
A_{\omega n} e=1}}\left\|\phi_{e}^{\prime}\right\|^{h^{*}} m_{k}\left(\phi_{\omega}\left(\phi_{e}\left(X_{e}\right)\right)\right)+\frac{1}{2} \sum_{\omega \in E_{k}^{n}}\left\|\phi_{\omega}^{\prime}\right\|^{h^{*}} \\
& \leq T_{q}^{-1} e^{Q h_{k}} \sum_{\omega \in E_{k}^{n}} \sum_{\substack{e \leq q \\
A_{\omega_{n} e=1}}}\left\|\phi_{e}^{\prime}\right\|^{h^{*}} m_{k}\left(\phi_{\omega}\left(X_{\omega}\right)\right)+\frac{1}{2} \sum_{\omega \in E_{k}^{n}}\left\|\phi_{\omega}^{\prime}\right\|^{h^{*}} \\
& =T_{q}^{-1} e^{Q h_{k}} \sum_{\substack{e \leq q \\
A_{\omega_{n} e=1}}}\left\|\phi_{e}^{\prime}\right\|^{h^{*}} \sum_{\omega \in E_{k}^{n}} m_{k}\left(\phi_{\omega}\left(X_{\omega}\right)\right)+\frac{1}{2} Z_{n}\left(h^{*}, k\right) \\
& =T_{q}^{-1} e^{Q h_{k}} \sum_{\substack{e \leq q \\
A_{\omega_{n} e=1}}}\left\|\phi_{e}^{\prime}\right\|^{h^{*}}+\frac{1}{2} Z_{n}\left(h^{*}, k\right) \\
& \leq T_{q}^{-1} e^{Q d} \Sigma_{h^{*}}+\frac{1}{2} Z_{n}\left(h^{*}, k\right) .
\end{aligned}
$$

A straightforward inductive argument then gives

$$
\begin{aligned}
Z_{n}\left(h^{*}, k\right) & \leq 2 \Sigma_{h^{*}} T_{q}^{-1} e^{Q d}+2^{-(n-1)} Z_{1}\left(h^{*}, k\right) \leq 2 \Sigma_{h^{*}} T_{q}^{-1} e^{Q d}+Z_{1}\left(h^{*}, \infty\right) \\
& =\Sigma_{h^{*}}\left(2 T_{q}^{-1} e^{Q d}+1\right)<\infty,
\end{aligned}
$$

where the last inequality follows from (4.8). Hence, we obtain

$$
\mathrm{P}\left(h^{*}\right)=\limsup _{n \rightarrow \infty} \frac{1}{n} \log Z_{n}\left(h^{*}, \infty\right) \leq \limsup _{n \rightarrow \infty} \frac{1}{n} \log \left(\Sigma_{h^{*}}\left(2 T_{q}^{-1} e^{Q d}+1\right)\right)=0 .
$$

It follows that $h^{*} \geq u$. By combining this with (4.10), we conclude that $h \leq u \leq h^{*}$. Since obviously $h^{*} \leq h$, we now obtain

$$
u=h^{*}=h .
$$

Finally, using (4.9) and Proposition 4.10, we conclude that $\mathrm{P}(h)=0$, and also that $h$ is the only zero of the pressure function $\mathrm{P}$. 


\section{Applichtions to infinitely Generated Schottky Group}

5.1. Preliminaries for Kleinian Limit Sets. Hyperbolic geometry in the upper-half-space model $\left(\mathbb{H}^{d+1}, d_{h y p}\right)$ and conformal geometry on the boundary $\partial \mathbb{H}^{d+1}=\mathbb{R}^{d} \cup\{\infty\}$ have the same automorphism group, namely the group $\operatorname{Isom}(d+1)$ of hyperbolic isometries is isomorphic to the group $\operatorname{Con}(d)$ of conformal maps in $\partial \mathbb{H}^{d+1}$. Therefore, any transformation in $\operatorname{Isom}(d+1)$ gives rise to a conformal map in $\partial \mathbb{H}^{d+1}$, and vice versa. It is well-known that this isomorphism arises naturally from the principle of Poincaré extension, based on the elementary observation that a $(d+1)$-dimensional hyperbolic half-space $H$ in $\mathbb{H}^{d+1}$ corresponds in a unique way to a $d$-dimensional ball $D:=\bar{H} \cap \partial \mathbb{H}^{d+1}$.

Recall that a Kleinian group $G$ is a discrete subgroup of Con $(d)$, and that the limit set $L(G)$ of $G$ is the derived set of some arbitrary $G$-orbit, that is $L(G):=\overline{G(z)} \backslash G(z)$, for some arbitrary $z \in \mathbb{H}^{d+1} \cup \partial \mathbb{H}^{d+1}$. It is well-known that $L(G)$ can always be decomposed into the set $L_{r}(G)$ of radial limit points and the set $L_{t}(G)$ of transient limit points, where

$$
L_{r}(G):=\left\{\xi \in L(G): \liminf _{T \rightarrow \infty} \Delta\left(\xi_{T}\right)<\infty\right\} \text { and } L_{t}(G):=\left\{\xi \in L(G): \lim _{T \rightarrow \infty} \Delta\left(\xi_{T}\right)=\infty\right\} .
$$

In here, $\xi_{T}$ refers to the point on the hyperbolic geodesic ray from $i_{d}:=(0, \ldots, 0,1) \in \partial \mathbb{H}^{d+1}$ to $\xi$ for which $d_{h y p}\left(i_{d}, \xi_{T}\right)=T$, and $\Delta\left(\xi_{T}\right)$ refers to the hyperbolic distance of $\xi_{T}$ to the orbit $G\left(i_{d}\right)$, that is $\Delta\left(\xi_{T}\right):=\inf \left\{d_{h y p}\left(\xi_{T}, g\left(i_{d}\right)\right): g \in G\right\}$. Important subsets of $L(G)$ are the set $L_{u r}(G)$ of uniformly radial limit points and the set $L_{J}(G)$ of Jørgensen limit points. These are given as follows (cf. [4] [10]).

$$
L_{u r}(G):=\left\{\xi \in L(G): \limsup _{T \rightarrow \infty} \Delta\left(\xi_{T}\right)<\infty\right\},
$$

$L_{J}(G):=\{\xi \in L(G)$ : there exists a geodesic ray towards $\xi$ which is

fully contained in some Dirichlet fundamental domain of $G$ \}.

One easily verifies that $L_{u r}(G) \subseteq L_{r}(G)$ and that $L_{J}(G) \subseteq L_{t}(G)$. We remark that in [1] it was shown for any arbitrary non-elementary Kleinian group $G$ that the Hausdorff dimension of $L_{u r}(G)$ is equal to the exponent of convergence $\delta(G)$ of the Poincaré series $\sum_{g \in G} \exp \left(-s d_{h y p}\left(i_{d}, g\left(i_{d}\right)\right)\right)$ associated with $G$ (see also [14]). Finally, we briefly comment on how $L_{J}(G)$ relates to some of the concepts used in the study of rigidity of Kleinian groups acting on $\mathbb{H}^{3}$ (for the proofs and further details we refer to [16] (see also [7])). With $F_{z}(G)$ referring to the closure in $\mathbb{R}^{3}$ of the Dirichlet polyhedron centred at $z \in \mathbb{H}^{3}$, let $F_{z}^{\infty}(G):=F_{z}(G) \cap \partial \mathbb{H}^{3}$. We then have that $F_{z}^{\infty}(G)$ is a wandering set for $G$, that is we have $\lambda_{2}\left(F_{z}^{\infty}(G) \cap g\left(F_{z}^{\infty}(G)\right)\right)=0$ for all $g \in G \backslash\{i d$. $\}$ (where $\lambda_{2}$ refers to the 2-dimensional Lebesgue measure). Note that if $\xi \in F_{z}^{\infty}(G)$ then the orbit $G(z)$ does not intersect the interior of the horoball at $\xi$ through $z$. Therefore, the Dirichlet set $D_{z}(G):=\bigcup_{\gamma \in G} g\left(F_{z}^{\infty}(G)\right)$ does not contain any horospherical limit points (recall that a point $\xi$ is called horospherical if every horoball at $\xi$ contains infinitely many elements of $G(z))$. One then immediately verifies that $L_{J}(G) \subseteq D_{z}(G)$. 
5.2. Infinitely Generated Schottky Groups of Rapid Decay and Discrepancy Type. We now restrict the discussion to a very special class of Kleinian groups which we will call infinitely generated classical Schottky groups of rapid decay. In order to define these groups, let $\mathcal{D}:=\left\{D_{n}^{i}: n \in \mathbb{N}, i \in\{0,1\}\right\}$ be an infinite set of pairwise disjoint open $d$-dimensional balls in $\partial \mathbb{H}^{d+1}$. For ease of exposition we assume that $\operatorname{diam}\left(D_{n}^{i}\right)=\operatorname{diam}\left(D_{n}^{i \oplus 1}\right)$ for all $n \in \mathbb{N}$, where " $\oplus$ " refers to addition modulo 2. Furthermore, let $\left\{g_{n}^{i}: n \in \mathbb{N}, i \in\{0,1\}\right\}$ be a set of hyperbolic transformations in $\operatorname{Con}(d)$ such that for all $n \in \mathbb{N}$ and $i \in\{0,1\}$,

$$
g_{n}^{i}\left(\operatorname{Ext}\left(D_{n}^{i}\right)\right)=\operatorname{Int}\left(D_{n}^{i \oplus 1}\right) \text { for all } n \in \mathbb{N}, i \in\{0,1\} .
$$

In here Int and Ext denote respectively the interior and exterior with respect to the Euclidean metric in $\mathbb{R}^{d}$. We will always assume that $\mathcal{D}$ satisfies the following rapid decay condition, where for $\alpha>0$ and for a ball $D \in \mathcal{D}$ of radius $r>0$ we let $\alpha D$ denote the ball of radius $\alpha r$ with the same centre as $D$.

Rapid Decay Condition. There exists an increasing sequence $\left(\alpha_{n}\right)_{n \in \mathbb{N}}$ of real numbers $\alpha_{n}>2$ such that $\lim _{n \rightarrow \infty} \alpha_{n}=\infty$ and

$$
\alpha_{n} D_{n}^{i} \cap \alpha_{m} D_{m}^{j}=\emptyset \text { for all distinct pairs }(n, i),(m, j) \in \mathbb{N} \times\{0,1\} .
$$

Finally, we always assume that there exists $R>0$ such that $\alpha_{n} D_{n}^{i} \subset B(0, R)$ for all $(n, i) \in$ $\mathbb{N} \times\{0,1\}$, where $B(0, R)$ refers to the closed $d$-dimensional ball with centre $0 \in \mathbb{R}^{d}$ and radius $R$. Then put $Y(G):=B(0,3 R)$. Now, let $G$ be the group generated by $\left\{g_{n}^{i}: n \in\right.$ $\mathbb{I}, i \in\{0,1\}\}$. One easily verifies that $\bigcap_{D \in \mathcal{D}} \operatorname{Ext}(D)$ is a fundamental domain for the action of $G$ on $\partial \mathbb{H}^{d+1}$ (cf. [5], Proposition VIII A.4). This then gives that $G$ is a Kleinian group, and we will refer to $G$ as an infinitely generated Schottky group of rapid decay. We remark that for $d=2$, by viewing the $g_{n}^{i}$ as elements of $\operatorname{Isom}(3)$, the set $\mathbb{H}^{3} / G$ of equivalence classes is topologically a 3-manifold whose interior is homeomorphic to a 3-dimensional ball to which infinitely many solid handles are attached, and whose boundary is the surface of that handlebody. We will now see that every infinitely generated Schottky group of rapid decay gives rise to some conformal pseudo-Markov system.

Theorem 5.1. Let $G$ be an infinitely generated Schottky group $G$ of rapid decay as defined above. Define $X_{n}^{i}:=Y(G) \backslash \alpha_{n} D_{n}^{i}$ and $\phi_{n}^{i}:=\left.g_{n}^{i}\right|_{X_{n}^{i}}$, for each $(n, i) \in \mathbb{N} \times\{0,1\}$. Put $E=E(G)=\mathbb{N} \times\{0,1\}\}$ and define the incidence matrix $A: E \times E \rightarrow\{0,1\}$ by $A_{(n, i)(m, j)}=$ 1 if and only if $(n, i) \neq(m, j \oplus 1)$. Then $\mathcal{S}(G):=\left\{\phi_{n}^{i}:(n, i) \in E\right\}$ is a conformal pseudoMarkov system.

Proof. Consider the system $\mathcal{S}(G)$ given as stated in the theorem, and note that by (5.1) we have

$$
\phi_{n}^{i}\left(X_{n}^{i}\right) \subset D_{n}^{i \oplus 1}
$$

One immediately verifies that $A$ is finitely irreducible (take for instance $\Xi:=\{(1,0),(1,1)\}$ ). In order to show that $\mathcal{S}(G)$ is a a conformal pseudo-Markov system, note that (b), (c) and (d) in the definition of a pseudo-Markov system are immediate consequences of (5.2) and (5.3). Also, (3) and (4) in the definition of a conformal pseudo-Markov system are trivially satisfied, 
and the pseudo-convexity in (1) of Definition 4.1 is satisfied with $K=\pi / 2$. In order to show that the Pre-Distortion Property (5) in Definition 4.1 is satisfied, let $(n, i),(m, j) \in E$ be fixed such that $A_{(n, i)(m, j)}=1$. By (5.3) we have $\phi_{m}^{j}\left(X_{m}^{j}\right) \subset D_{m}^{j \oplus 1} \subset B(0,2 R)$. Since $\mathbb{R}^{d} \backslash X_{n}^{i} \subseteq\left(\mathbb{R}^{d} \backslash Y(G)\right) \cup \alpha_{n} D_{n}^{i}$, since $(n, i) \neq(m, j \oplus 1)$ and since $\alpha_{n}>2$, (5.2) implies that

$$
\operatorname{dist}\left(\phi_{m}^{j}\left(X_{m}^{j}\right), \mathbb{R}^{d} \backslash X_{n}^{i}\right) \geq \alpha_{m} \frac{\operatorname{diam}\left(\phi_{m}^{j}\left(X_{m}^{j}\right)\right)}{2}>\operatorname{diam}\left(\phi_{m}^{j}\left(X_{m}^{j}\right)\right) .
$$

Hence, we can apply Lemma 4.3 with $\kappa=1$, which gives that the system $\mathcal{S}(G)$ has the PreDistortion Property. In order to verify the remaining properties, let $(n, i) \in \mathbb{N} \times\{0,1\}$ be fixed. We now first give an upper estimate for the conformal derivative of $\phi_{n}^{i}$. Note that we can assume without loss of generality that $i_{d}=(0, \ldots, 0,1) \in \mathbb{H}^{d+1}$ is not contained in the Poincaré extensions of the balls in $\left\{D_{k}^{j}:(k, j) \in \mathbb{N} \times\{0,1\}\right\}$. By recalling that the conformal derivative is given by the Poisson kernel and then using elementary Euclidean and hyperbolic geometry, we obtain for each $z \in \operatorname{Ext}\left(\alpha_{n} D_{n}^{i}\right)$,

$$
\begin{aligned}
\left|\left(g_{n}^{i}\right)^{\prime}(z)\right| & =\frac{p_{d+1}\left(\left(g_{n}^{i}\right)^{-1}\left(i_{d}\right)\right)}{\left|z-\left(g_{n}^{i}\right)^{-1}\left(i_{d}\right)\right|^{2}} \leq \frac{p_{d+1}\left(\left(g_{n}^{i}\right)^{-1}\left(i_{d}\right)\right)}{\left(\left(\alpha_{n} \operatorname{diam}\left(D_{n}^{i}\right)-\operatorname{diam}\left(D_{n}^{i}\right)\right) / 2\right)^{2}} \\
& \leq \frac{1}{\left(\alpha_{n}-1\right)^{2}} \frac{p_{d+1}\left(\left(g_{n}^{i}\right)^{-1}\left(i_{d}\right)\right)}{\left(\operatorname{diam}\left(D_{n}^{i}\right) / 2\right)^{2}} \leq \frac{1}{\left(\alpha_{n}-1\right)^{2}} .
\end{aligned}
$$

In here $p_{d+1}: \mathbb{H}^{d+1} \rightarrow \mathbb{R}^{+}$refers to the projection onto the $(d+1)$-th coordinate. From this we deduce that

$$
\left\|\left(\phi_{n}^{i}\right)^{\prime}\right\| \leq\left(\alpha_{n}-1\right)^{-2}<4 \alpha_{n}^{-2} .
$$

This formula immediately implies that conditions (2) in Definition 4.1 and (a) in Definition 3.1 are satisfied. This finishes the proof of the theorem.

One immediately verifies that for an infinitely generated Schottky group $G$ of rapid decay there is the following dictionary translating the most important subsets of the limit set of $G$ into the corresponding subsets of the limit set of the associated conformal pseudo-Markov system $\mathcal{S}(G)$. In here, $\mathcal{S}_{D}(G)$ refers to the subsystem of $\mathcal{S}(G)$ giving rise to all admissable words built from the finite alphabet $D \subset E(G)$.

$$
\begin{aligned}
& L(G)=\overline{J_{\mathcal{S}(G)}} ; \\
& L(G) \backslash L_{J}(G)=J_{\mathcal{S}(G)} ; \\
& L_{u r}(G)=\bigcup_{D \subset E(G) \text { finite }} J_{\mathcal{S}_{D}(G)} \\
& L_{J}(G)=\bigcup_{\omega \in E_{A}^{*}} \phi_{\omega}\left(\Delta(\mathcal{S}(G)) \cap X_{\omega}\right) .
\end{aligned}
$$

We therefore have in particular $h^{*}(\mathcal{S}(G))=\operatorname{HD}\left(L_{u r}(G)\right)$ and $h(\mathcal{S}(G))=\operatorname{HD}\left(L(G) \backslash L_{J}(G)\right)$. Hence, by combining Theorem 5.1 and Theorem 4.13, we obtain the following corollary. 
Corollary 5.2. Let $G$ be an infinitely generated Schottky group $G$ of rapid decay such that the associated conformal pseudo-Markov system $\mathcal{S}(G)$ is thin. We then have

$$
\delta(G)=\operatorname{HD}\left(L_{u r}(G)\right)=h^{*}(\mathcal{S}(G))=h(\mathcal{S}(G))=\operatorname{HD}\left(L(G) \backslash L_{J}(G)\right) .
$$

Apriori, the existence of infinitely generated Schottky groups as considered in the latter corollary is not clear. In fact, this will follow from our final theorem in which we obtain the existence of a very special type of infinitely generated Schottky groups of rapid decay fulfilling the thinness condition. Before stating this theorem, let us recall the notion of a discrepancy group, introduced in [4]. Namely, a Kleinian group $G$ is called discrepancy group if and only if $\operatorname{HD}\left(L_{r}(G)\right)<\operatorname{HD}(L(G))$. Kleinian groups of this type were first shown to exist by Patterson in [12], Theorem 4.4 (see also [11] [13]). (For further discussions and examples of Kleinian discrepancy groups we refer to [3] [4] [6]). We remark that the discrepancy groups considered in [12] and [13] are Schottky groups of the first kind, that is their limit set is the whole boundary of the underlying hyperbolic space. Therefore, a natural question to ask is if there are Schottky discrepancy groups which are of the second kind. A particular outcome of the following theorem will be to give an affirmative answer to this question. Also, note that a Kleinian group $G$ considered in the following theorem has the remarkable property that $\operatorname{HD}\left(L_{u r}(G)\right)=\operatorname{HD}\left(L(G) \backslash L_{J}(G)\right)$. Therefore, a further particular outcome of the following theorem is that for discrepancy groups we can not expect in general that the dimension gap between $L_{u r}(G)$ and $L(G)$ can be filled smoothly by subsets of $L(G) \backslash L_{J}(G)$.

Theorem 5.3. For all $0<s<t \leq d$ there exists an infinitely generated Schottky group $G<\operatorname{Con}(d)$ of rapid decay such that $G$ is a discrepancy group with $\delta(G) \leq s<t=\operatorname{HD}(L(G))$. Furthermore, the conformal pseudo-Markov system associated with $G$ fulfills the thinness property, and therefore,

$$
\delta(G)=\operatorname{HD}\left(L_{u r}(G)\right)=\operatorname{HD}\left(L(G) \backslash L_{J}(G)\right)<\operatorname{HD}\left(L_{J}(G)\right)=\operatorname{HD}(L(G))=t .
$$

Proof. The proof consists of giving the explicit construction of an infinitely generated Schottky group $G<\operatorname{Con}(d)$ of rapid decay which has the properties as stated in the theorem. Suppose that $W \subset \mathbb{R}^{d}$ is some compact set such that $\operatorname{HD}(W)=t$ and such that there exists a countable set $V=\left\{v_{n}^{i}: n \in \mathbb{N}, i \in\{0,1\}\right\} \subset \mathbb{R}^{d} \backslash W$ whose derived set is equal to $W$, that is $W=\bar{V} \backslash V$. Also, let $\left(\alpha_{n}\right)_{n \in \mathbb{N}}$ be some increasing sequence of real numbers $\alpha_{n}>2$ such that

$$
\sum_{n \in \mathbb{N}} \alpha_{n}^{-2 s}<\frac{1}{4} \text { and } \sum_{n \in \mathbb{N}} \alpha_{n}^{-\theta}<\infty \text { for all } \theta>0 .
$$

Furthermore, fix some decreasing sequence $\left(\epsilon_{n}\right)_{n \in \mathbb{N}}$ of positive numbers such that

$$
\lim _{n \rightarrow \infty} \alpha_{n} \epsilon_{n}=0 \text { and } \sup \left\{\alpha_{n} \epsilon_{n}: n \in \mathbb{N}\right\} \leq 1 .
$$


We will now construct a sequence $\left\{D_{n}^{i}: n \in \mathbb{N}, i \in\{0,1\}\right\}$ of $d$-dimensional open balls in $\mathbb{R}^{d}$ by induction as follows. For $n=1$ and $i \in\{0,1\}$, choose $D_{1}^{i}$ to be centred at $v_{1}^{i}$ such that

$$
V \cap \alpha_{1} D_{1}^{i}=\left\{v_{1}^{i}\right\}, \alpha_{1} D_{1}^{0} \cap \alpha_{1} D_{1}^{1}=\emptyset \text { and } \operatorname{diam}\left(D_{1}^{0}\right)=\operatorname{diam}\left(D_{1}^{1}\right) \leq \epsilon_{1} .
$$

For the inductive step suppose that the $2 n$ balls $\left\{D_{k}^{i}: 1 \leq k \leq n, i \in\{0,1\}\right\}$ have already been constructed. For each $i \in\{0,1\}$, we then choose the ball $D_{n+1}^{i}$ to be centred at $v_{n+1}^{i}$ such that $V \cap \alpha_{n+1} D_{n+1}^{i}=\left\{v_{n+1}^{i}\right\}$ and

$$
\alpha_{n+1} D_{n+1}^{0} \cap \alpha_{n+1} D_{n+1}^{1}=\emptyset, \alpha_{n+1} D_{n+1}^{i} \cap \alpha_{k} D_{k}^{j}=\emptyset
$$

for all $1 \leq k \leq n, j \in\{0,1\}$, as well as

$$
\operatorname{diam}\left(D_{n+1}^{0}\right)=\operatorname{diam}\left(D_{n+1}^{1}\right) \leq \epsilon_{n+1} .
$$

We then choose the set $\left\{g_{n}^{i}: n \in \mathbb{N}, i \in\{0,1\}\right\}$ of hyperbolic elements of $\operatorname{Con}(d)$ such that

$$
g_{n}^{i}\left(\operatorname{Ext}\left(D_{n}^{i}\right)\right)=\operatorname{Int}\left(D_{n}^{i \oplus 1}\right) \text { for all } n \in \mathbb{N}, i \in\{0,1\} \text {. }
$$

One immediately verifies that the group $G$ generated by $\left\{g_{n}^{i}: n \in \mathbb{N}, i \in\{0,1\}\right\}$ is an infinitely generated Schottky group of rapid decay. (Note that the conditions in (5.6) are necessary to ensure that $Y(G)$ is bounded). By Theorem 5.1 we then have that $G$ gives rise to a conformal pseudo-Markov system $\mathcal{S}(G)$ such that $\overline{J_{\mathcal{S}(G)}}=L(G)$. Furthermore, by combining (5.4) with the second condition in (5.5), we obtain that $\sum_{n, i}\left\|\phi_{n}^{i}\right\|^{\theta}$ converges for every $\theta>0$. So, the system $\mathcal{S}(G)$ is thin. Combining in turn the first part of (5.5), (5.4) (which both imply that $\mathrm{P}(s)<0)$, Proposition 4.10 (a) and the first part of Theorem 4.13, we obtain that $\delta(G)=\operatorname{HD}\left(J_{\mathcal{S}(G)}\right) \leq s$. Since $L_{J}(G)=\bigcup_{\omega \in E_{A}^{*}} \phi_{\omega}\left(\Delta(\mathcal{S}(G)) \cap X_{\omega}\right)$ and since by construction $\Delta(\mathcal{S}(G))=W$, we obtain that $\operatorname{HD}\left(L_{J}(G)\right)=\operatorname{HD}(W)=t$. Combining these observations with Proposition 4.4, it follows that $\operatorname{HD}(L(G))=\operatorname{HD}\left(L_{J}(G)\right)=t>s \geq \operatorname{HD}\left(J_{\mathcal{S}(G)}\right)=\delta(G)$. This finishes the proof of the theorem.

\section{REFERENCES}

[1] C. J. Bishop, P. W. Jones, 'Hausdorff dimension and Kleinian groups', Acta Math. 56 (1997) 1-39.

[2] R. Bowen, 'Equilibrium states and the ergodic theory for Anosov diffeomorphisms', Lect. Notes in Math. 470, Springer, 1975.

[3] R. Brooks, 'The bottom of the spectrum of a Riemannian covering', J. Reine Angew. Math. 357 (1985) 101-114.

[4] K. Falk, B.O. Stratmann, 'Remarks on Hausdorff dimensions for transient limit sets of Kleinian groups', Tohoku Math. J. (2), 56 (4) 571-582.

[5] B. Maskit, Kleinian groups, Springer Verlag, Berlin, 1989.

[6] K. Matsuzaki, 'The Hausdorff dimension of the limit sets of infinitely generated Kleinian groups', Math. Proc. Cambridge Philos. Soc. 128 (2000) 123-139.

[7] K. Matsuzaki and M. Taniguchi, Hyperbolic manifolds and Kleinian groups, Oxford Math. Monographs, 1998.

[8] D. Mauldin, M. Urbański, 'Gibbs states on the symbolic space over an infinite alphabet', Israel. J. of Math. 125 (2001) 93-130. 
[9] R. D. Mauldin, M. Urbański, Graph directed Markov systems: geometry and dynamics of limit sets, Cambridge Univ. Press, 2003.

[10] J. R. Parker, B. O. Stratmann, 'Kleinian groups with singly cusped parabolic fixed points', Kodai J. Math. 24 (2001) 169-206.

[11] S. J. Patterson, 'The exponent of convergence of Poincaré series', Monatsh. Math. 82 (1976) 297315.

[12] S. J. Patterson, 'Some examples of Fuchsian groups', Proc. London Math. Soc. (3) 39 (1979) 276-298.

[13] S. J. Patterson, 'Further remarks on the exponent of convergence of Poincaré series', Tohoku Math. J. 35 (1983) 357-373.

[14] B. O. Stratmann, 'The exponent of convergence of Kleinian groups; on a theorem of Bishop and Jones', in Progress in Probability (Birkhäuser Verlag) Vol. 57 (2004) 93-107.

[15] B. O. Stratmann, 'Fractal geometry on hyperbolic manifolds', Mathematica Gottingensis 09 (2003) 1-20; to be published in Proceedings of the 'Janos Bolyai Conference on Hyperbolic Geometry' (Budapest, 2002); Kluwer Acad. Publ..

[16] D. Sullivan, 'On the ergodic theory at infinity of an arbitrary discrete group of hyperbolic motions', Riemann surfaces and related topics, Ann. of Math. Stud. 97 (1981) 465-496.

Bernd O. Stratmann, Department of Mathematics, University of St Andrews, St Andrews KY16 9SS, SCOTLAND

E-MAIL: BOSMATHS.ST-AND.AC.UK

WEB:HTTP://WWW-MATHS.MCS.ST-ANDREWS.AC.UK/ BOS/

Mariusz Urbański, Department of Mathematics, University of North Texas, P.O. Box 311430, DENTON, TX 76203-1430, USA

E-MAIL:URBANSKIUNT.EDU

WEB: HTTP://WWW.MATH.UNT.EDU/ URBANSKI 